\title{
Microscopic modelling of the flow properties of polymers
}

\author{
R J J Jongschaap \\ University of Twente, Department of Applied Physics, Centre for Rheology, Enschede, The Netherlands
}

\begin{abstract}
The understanding of the flow behaviour of polymeric liquids is of great interest from a practical as well as a theoretical point of view. An important part of the research in this field consists of the development of suitable models, describing the rheological properties of the materials. Depending upon its purpose, such a model may be based upon empirical knowledge of the macroscopical flow behaviour or on information about the microstructure of the materials. Moreover, for a given system, different types of modelling may be possible. In order to provide an overview of the various approaches in this area the basic principles of some important models are discussed: continuum, bead-rod-spring, transient network, reptation and configuration tensor models. Emphasis has been put on a consistent treatment of the fundamentals of the various models and their interrelationship, rather than considering any of them in much detail.
\end{abstract}

This review was received in August 1989. 


\section{Contents}

1. Introduction

2. Continuum mechanics

2.1. Introduction

2.2. Simple fluid theory

2.3. Special flows

2.4. Special constitutive equations

3. Elements of modelling

3.1. Introduction

3.2. Structure

3.3. The average stress tensor

4. Bead-rod-spring models

4.1. Introduction

4.2. Examples

5. Transient-network models

5.1. Introduction

5.2. The Lodge model

5.3. Generalisations

6. Reptation models

6.1. Introduction

6.2. Tube models

6.3. The Curtiss-Bird model

7. Generalised continuum theories 41

7.1. Introduction

7.2. Configuration tensor models

7.3. Applications

8. Discussion

Appendix 1. Frames of reference

Appendix 2. Convected derivatives 


\section{Introduction}

The understanding of the flow behaviour of polymeric liquids is important since these systems are used in many industrial processes. Moreover they are also present in many biological systems. So, from a practical point of view there is a great need for research in this area. From a purely scientific point of view, polymeric liquids are also very interesting, since on one hand they show a flow behaviour with striking qualitative differences from ordinary liquids, while on the other hand they possess a well defined and relatively simple microstructure.

The flow behaviour of polymeric systems is a very extensive and complicated area. At present it is even an important part of a separate branch of science called rheology (the science of deformation and flow of materials). In the present review we will treat a part of this field in some detail. First, however, we will now give a somewhat broader overview.

In the processing and application of polymeric liquids one has to deal with flow problems, as in ordinary fluid mechanics. The difference from ordinary fluid mechanics, however, is that the differential equation by which the flow is determined is no longer the Navier-Stokes equation but a far more complicated equation or set of equations which may be different for different polymers. Moreover, whereas in ordinary fluid mechanics the fluid properties are completely specified by the value of one parameter, the viscosity, in the case of polymeric liquids one has to specify a number of material parameters or functions. Even if this has been done, the solution of flow problems is a complicated task which in most cases has to be treated numerically. In the present review we will not deal with this subject. For an introduction we refer to the textbooks of Crochet et al (1984), Tanner (1985) and Pearson (1985).

The reason that the flow equations may be different for different materials is the differences in the underlying constitutive equations. These equations reflect the specific mechanical properties of the material. Well known examples are Newton's law for viscous fluids and Hooke's law for elastic materials. A central problem in polymer rheology is the determination of adequate constitutive equations for polymeric systems. In the study of these equations one does not consider complicated flow situations, but certain simple and well defined flow geometries in which critical tests on the validity of constitutive equations are possible. In such experiments the values of material parameters and functions in the constitutive equations may also be measured. The branch of rheology which is concerned with these types of measurements is called rheometry. For an introduction we refer to the book of Walters (1965) on this subject.

The development of constitutive equations may be based upon continuum mechanics. This method offers the possibility of combining general results about the shape of constitutive equations, based upon general principles, with empirical results obtained from rheological measurements. As a result one may arrive at useful forms of the constitutive equations. In $\$ 2$ the principles of continuum mechanics and the macroscopic theory of constitutive equations will be discussed. For further reference we refer to the textbook by Bird et al (1987a) and the book of Astarita and Marrucci (1974).

A problem with the continuum approach is that even if one arrives at a constitutive equation which properly describes the rheometrical data it is not possible to relate the 
parameters of that equation to molecular properties or other aspects of the microstructure of the system. Furthermore it is hard to obtain specific information about the constitutive equation from macroscopic considerations alone. In order to solve these problems theories have been developed, based upon our knowledge of the molecular structure of polymeric liquids. At first, these theories were concerned with particular types of rheological behaviour like linear viscoelasticity and simple shear flow, but later on attention was also directed to the derivation of full constitutive equations. At present, both directions are of interest. By restricting to one particular type of flow it is possible to retain more details about the microstructure. This is of interest in cases where rheological measurements are used to measure molecular quantities. The derivation of the total constitutive equation from molecular considerations is primarily of interest in relation to the macroscopical theories based upon continuum mechanics. The main part of the present review is also devoted to molecular theories. We will treat this subject with emphasis on the basic structure of each theory rather than by considering many details and special cases. We will also concentrate on a particular aspect, namely micro-rheological modelling, i.e. the use of more or less artificial structures in order to represent the real microstructure. A model may be considered as an image of the real system, simplified in such a way that it is tractable to mathematical analysis and still represents features of the system that are expected to be important for its behaviour.

Important steps in the process of modelling are: the definition of the model structure; its evolution in time; and the relation between microscopic forces and the macroscopic stress tensor. These items and some general aspects of micro-rheological modelling will be discussed in $\S 3$.

In $\S \S 4-7$ three important special classes of models will be discussed in some detail. These are bead-rod-spring models, transient-network models, reptation models and configuration tensor models.

We will not discuss the various special cases of each type of model, but rather treat some representative examples. We will also not consider in detail the predictions of the rheological behaviour of the various models. For such more detailed information we refer to textbooks on the subject (e.g. Doi and Edwards (1986), Bird et al (1987b), Larson (1988)). Instead, in the present review, we will try to give a consistent treatment of the principles of each of the types of model in order to obtain an overview and to gain insight into the possibilities of applying, combining or extending the various approaches. In $\S 8$ we will return to this point.

\section{Continuum mechanics}

\subsection{Introduction}

The main part of the present review deals with microscopical theories of the rheological behaviour of polymeric liquids. First, however, we will consider the underlying macroscopical framework. Without such a framework it would hardly be possible to formulate general microscopical theories of the flow behaviour of polymeric liquids. Indeed, in the historical development we see the important impact on those molecular theories of the modern formulation of continuum mechanics and the macroscopical theories of constitutive equations.

Important achievements in this respect were a theory on constitutive equations by Oldroyd (1950) and the famous theory on 'simple materials' by Noll (1958). In the 
present review we will follow the treatment of Noll and especially his so called 'simple fluid theory'. For an extensive treatment we refer to the article of Truesdell and Noll (1965).

The theory will be outlined in the following parts: first $(\$ 2.2)$ the description of deformation and stresses in a continuum and the general formulation of constitutive equations, then (in $\S 2.3$ ) some special types of flow and the associated rheological material functions and finally $(\$ 2.4)$, some special constitutive equations.

\subsection{Simple fluid theory}

We start our discussion of simple fluid theory with a brief treatment of some kinematical notions. First we note that the deformation history of a continuum is fully specified by the function $\boldsymbol{\xi}=\boldsymbol{\xi}_{\kappa}(\boldsymbol{X}, \tau)$. Here $\boldsymbol{\xi}$ is the position at time $\tau$ of a particle which is located at a position $\boldsymbol{X}$ in an arbitrary reference configuration $\kappa$. (In this section by 'particle' we mean a material point in the continuum.)

Often, especially for fluids, the configuration at the 'present' time $t$ is chosen as a reference configuration. In this, so called, relative description the deformation function becomes

$$
\boldsymbol{\xi}=\xi_{t}(x, \tau) .
$$

The gradient of this function

$$
F_{t}(\tau)=\frac{\partial \boldsymbol{\xi}_{t}}{\partial \boldsymbol{x}}
$$

is called the relative deformation gradient. On this tensor several measures of deformation are based. For later use we mention the polar decomposition of $F_{t}$ in an orthogonal 'rotation tensor' $\boldsymbol{R}_{t}$ and the positive definite and symmetric 'stretch tensors' $\boldsymbol{U}_{i}$ and $\boldsymbol{V}_{r}$

$$
\boldsymbol{F}_{t}=\boldsymbol{R}_{t} \cdot \boldsymbol{U}_{t}=\boldsymbol{V}_{t} \cdot \boldsymbol{R}_{t}
$$

Furthermore we will use the strain tensors of Cauchy and Finger, given by

$$
\boldsymbol{C}_{t}=\boldsymbol{F}_{t}^{\mathrm{T}} \cdot \boldsymbol{F}_{t} \quad \text { and } \quad \boldsymbol{C}_{t}^{-1}=\boldsymbol{F}_{t} \cdot \boldsymbol{F}_{t}^{-\mathrm{T}}
$$

respectively.

In the case of small deformations it is often useful to consider the field of displacement vectors $\boldsymbol{u}_{i}(\tau)=\boldsymbol{\xi}-\boldsymbol{x}$ and the displacement gradient tensor

$$
H_{t}=\frac{\partial u_{t}}{\partial \boldsymbol{x}}=F_{1}-1
$$

The symmetrical part

$$
\boldsymbol{E}_{t}=\frac{1}{2}\left(\boldsymbol{H}_{t}+\boldsymbol{H}_{t}^{\mathbf{T}}\right)
$$

is the (relative) infinitesimal strain tensor. In deformations in which $\boldsymbol{H}_{t}$ is small in the sense that $\left|\boldsymbol{H}_{t}\right| \ll 1$ the strain tensors $\boldsymbol{C}_{t}$ and $\boldsymbol{C}_{t}^{-1}$ may be approximated as

$$
C_{t} \simeq 1+2 E_{t} \quad \text { and } \quad C_{t}^{-1} \simeq 1-2 E_{t} \text {. }
$$

By taking the time derivative with respect to $\tau$ for $\tau=t$ of the relative deformation function (2.1) we obtain the velocity field $v(x, t)$. The gradient of this field will be denoted by

$$
\boldsymbol{L}=\frac{\partial \boldsymbol{v}}{\partial \boldsymbol{x}}=(\boldsymbol{\nabla} \boldsymbol{v})^{\mathrm{T}}
$$


The symmetric part of $L$ is the rate of strain tensor

$$
\boldsymbol{D}=\frac{1}{2}\left(\boldsymbol{L}+\boldsymbol{L}^{\mathrm{T}}\right)
$$

and the skew symmetrical part, the vorticity tensor

$$
\boldsymbol{W}=\frac{1}{2}\left(\boldsymbol{L}-\boldsymbol{L}^{\mathrm{T}}\right)
$$

Among the various higher-order rates of strain the most well known are the RivlinEricksen tensors:

$$
A_{\kappa}=\left.\frac{\mathrm{d}}{\mathrm{d} \tau} C_{i}(\tau)\right|_{\tau=i}
$$

In particular $\boldsymbol{A}_{1}=2 \boldsymbol{D}$.

We will now consider the description of the state of stress in a continuum. According to a fundamental theorem of Cauchy this is specified completely by a second-order tensor field $T(x, t)$. The tensor $T$, called the stress tensor, determines the traction $t$ on an arbitrary surface element $d S$ in the continuum by the relation $t=T \cdot n$ in which $n$ is the unit normal on the surface element. The stress tensor field is governed by balances of momentum and of moment of momentum. The former leads to the equation of motion

$$
\rho \dot{v}=\operatorname{div} T+\rho b
$$

in which $\rho$ is the mass density, $\dot{v}$ the acceleration field and $b$ an external body force field (per unit mass). The latter implies for an ordinary continuum the symmetry of the stress tensor: $T=T^{\mathrm{T}}$.

In the present review we will only consider incompressible materials. In that case, due to the incompressibility constraint, the stress tensor will contain a part that cannot contribute to the stress power in any deformation compatible with the constraint. This part may be proved to be an isotropic tensor, so we have

$$
T=p 1+T_{\mathrm{E}}
$$

The scalar $p$ in this expression is often called the undetermined pressure, since in a given deformation history its value is not determined by a constitutive equation. Instead, in a specific flow problem, the value of $p$ is determined by the equation of motion (2.12) and the boundary conditions. The quantity $\boldsymbol{T}_{\mathrm{E}}$ in (2.13), called the extra stress tensor, is the part of the stress tensor that for a given flow history is determined by a constitutive equation. If no particular flow problem is specified, the pressure term in (2.13) is undetermined, so the extra stress $T_{\mathrm{E}}$ is determined only up to an arbitrary isotropic term. This may be used to simplify the formulation of constitutive equations.

We now arrive at the main subject of this section: the simple fluid theory. For a thorough discussion of this theory, we again refer to the original papers of Noll (1958) and Truesdell and Noll (1965). Here we present only a brief outline for the case of incompressible fluids.

A simple material is defined (Noll 1958) as a material in which the extra stress tensor is a functional of the history of the deformation gradient evaluated at a fixed particle of the continuum. In the incompressible case we have

$$
T_{\mathrm{E}}=\mathscr{\mathscr { F }}_{\boldsymbol{\tau}-\infty}^{t}\left\{\boldsymbol{F}_{\kappa}(\tau)\right\}
$$


The index $\boldsymbol{\kappa}$ in the functional $\mathscr{F}^{\prime \prime}$ and the deformation gradient $\boldsymbol{F}$ denotes a dependence upon the reference configuration. A fluid is defined (Noll 1958) as a substance with such a high degree of symmetry that the constitutive equation is independent of the choice of the reference configuration. In that case the functional $\mathscr{F}_{\kappa}^{\prime \prime}$ in (2.14) becomes independent of $\kappa$ and instead of $\mathscr{F}_{\kappa}(\tau)$ the relative deformation gradient (2.2) may be used. So we obtain

$$
T_{\mathrm{E}}=\mathscr{F}^{\prime}\left\{\boldsymbol{F}_{t}(\tau)\right\} \text {. }
$$

The next step is the application of the principle of material frame indifference (Noll 1958). This means that equation (2.15) has to be invariant under a particular class of transformations $T \rightarrow T^{*}, F_{t}(\tau) \rightarrow F_{t}(\tau)^{*}$ called changes of frame. So for arbitrary changes of frame we have

$$
T_{\mathrm{E}}=\underset{\tau=-\infty}{\stackrel{t}{\mathscr{F}^{\prime}}}\left\{\boldsymbol{F}_{t}(\tau)\right\} \leftrightarrow T_{\mathrm{E}}^{*}=\underset{\tau=-\infty}{\stackrel{\prime}{\mathscr{F}^{\prime}}}\left\{\boldsymbol{F}_{t}^{*}(\tau)\right\}
$$

in which the functional $\mathscr{F}^{\prime}$ remains the same. The physical significance of a change of frame is a change from one observer to another one with a different position and orientation and a given relative motion with respect to the first one (see Appendix 1). So the requirement (2.16) upon the constitutive equation implies that such an equation should be invariant under arbitrary translations and rotations in space.

From (2.16) it may be proved (see Appendix 1) that the constitutive equation (2.15) reduces to

$$
T_{\mathrm{E}}=\underset{\tau=-\infty}{\mathscr{F}}\left\{C_{t}(\tau)\right\}
$$

in which $C_{t}(\tau)$ is the relative Cauchy deformation tensor defined by (2.4). From the principle of material frame indifference it also follows that the functional $\mathscr{F}$ in (2.17) is isotropic, i.e.

$$
\underset{\tau=-\infty}{\grave{F}}\left\{Q \cdot C_{t}(\tau) \cdot Q^{\mathrm{T}}\right\}=Q \cdot \underset{\tau=-\infty}{\mathscr{F}}\left\{C_{r}(\tau)\right\} \cdot Q^{\mathrm{T}}
$$

for arbitrary constant orthogonal tensors $Q$.

A direct consequence of $(2.18)$ is that under static conditions, i.e. at the 'rest history', $C_{t}(\tau)=1(-\infty<\tau<1)$, the extra stress becomes of the form $T_{\mathrm{E}}=-p 1$. So in simple fluid theory the usual property of a fluid of being a substance unable to maintain shear stresses at rest is a consequence of the definition (2.15) and the principle of material frame indifference.

The next principle by which a further reduction of the general constitutive equation (2.17) is possible is the principle of fading memory (Coleman and Noll (1961)). This principle expresses the physical experience that large, fast and/or recent deformations have more influence upon the present stress than deformations that are small, slow and/or less recent. It is possible to state this property in a precise mathematical form, by defining a norm $\|\cdot\|$ in the space of deformation histories, such that deformation histories which are expected to have a small (large) contribution to the present strain will have a small (large) magnitude corresponding to that norm.

The most important result that can be obtained from the principle of fading memory is an expansion for histories close to the rest history, in the sense that $\left\|C_{t}(\tau)-1\right\|$ is small $(-\infty<\tau<1)$. Under certain smoothness assumptions Coleman and Noll (1961) obtained an expansion of the functional (2.17) in a series of polynomial functionals. 
Under certain further assumptions of a rather formal mathematical nature this expansion can be transformed into a multiple integral expansion, originally proposed by Green and Rivlin (1957). In a somewhat simplified form, derived by Pipkin (1964), this expansion reads

$$
\begin{aligned}
T_{\mathrm{e}}=\int_{-\infty}^{t} \mu(t-\tau)\left(C_{t}(\tau)-1\right) \mathrm{d} \tau \\
\quad+\int_{-\infty}^{t} \int_{-\infty}^{t} \nu\left(t-\tau_{1}, t-\tau_{2}\right)\left(C_{t}\left(\tau_{1}\right)-1\right) \cdot\left(C_{t}\left(\tau_{2}\right)-1\right) \mathrm{d} \tau_{1} \mathrm{~d} \tau_{2}+\ldots
\end{aligned}
$$

where the remainder term goes to zero faster than $\left\|C_{r}(\tau)-1\right\|^{n}$ if the expansion is taken up to a $n$-tuple integral.

\subsection{Special flows}

In some special types of flow a further reduction of the general constitutive equation (2.17) or the integral expansion (2.19) is possible without making any further assumptions about specific properties of the fluid. Three properties of the flow are important in this context: (i) the magnitude of the deformations, (ii) the rates of deformation and (iii) the complexity of the flow geometry. If any of these properties is of a particular type, considerable simplification of the constitutive equation is possible.

In the case of small deformations the norm $\left\|C_{t}(\tau)-1\right\|$ will be small and only the first term in the integral expansion (2.19) remains significant. Moreover, on using (2.7), the tensor $C_{t}(\tau)$ may be expressed by the infinitesimal strain tensor $E_{t}$ and (2.19) becomes

$$
\boldsymbol{T}_{\mathrm{E}}=2 \int_{-\infty}^{t} \mu(t-\tau) \boldsymbol{E}_{t}(\tau) \mathrm{d} \tau
$$

This is the classical expression of linear viscoelasticity for the case of an incompressible fluid. So we see that for small deformations an explicit reduced form of the general constitutive equation (2.17) may be obtained. In this expression the material properties are fully described by one function, the so-called memory function $\mu(t)$. This function is closely related to the relaxation function $G(t)$, describing the material response upon a sudden step in strain. This can be seen by considering a step-strain history

$$
E_{t}(\tau)=\left\{\begin{array}{rl}
-E & \text { for } \tau<0<t \\
0 & \text { for } 0<\tau<t
\end{array} .\right.
$$

The stress response then becomes $\boldsymbol{T}_{\mathrm{E}}=2 G(t) \boldsymbol{E}$ in which $G(t)$ is the relaxation function. From (2.20) and (2.21) we see that

$$
G(t)=-\int_{1}^{\infty} \mu(s) \mathrm{d} s \text { or } \mu(t)=\frac{\mathrm{d}}{\mathrm{d} t} G(t) .
$$

Besides the relaxation function many other functions are used in specifying the material properties in linear viscoelasticity. For a discussion of these functions and their interrelationships we refer to textbooks like the one by Ferry (1980).

Here we just mention the complex dynamic modulus $G^{*}$, defined by

$$
\hat{T}=G^{*} \hat{E}
$$

in which $\hat{T}$ is the complex stress response upon the harmonic strain

$$
\hat{\boldsymbol{E}}_{t}(\tau)=\boldsymbol{E}\left(\mathrm{e}^{\mathrm{i} \omega \tau}-\mathrm{e}^{i \omega t}\right) \equiv \hat{\boldsymbol{E}}\left(\mathrm{e}^{i \omega(\tau-t)}-1\right)
$$


with $\hat{\boldsymbol{E}} \equiv \boldsymbol{E} \exp (\mathrm{i} \omega t)$. The real and imaginary parts $G^{\prime}(\omega)$ and $G^{\prime \prime}(\omega)$ of $G^{*}$ are called the storage modulus and the loss modulus, respectively. By substitution of (2.24) in the integral expression (2.20) and using (2.23) it may be proved that

$$
\begin{aligned}
& G^{\prime}(\omega)=\omega \int_{0}^{\infty} G(s) \sin \omega s \mathrm{~d} s \\
& G^{\prime \prime}(\omega)=\omega \int_{0}^{\infty} G(s) \cos \omega s \mathrm{~d} s .
\end{aligned}
$$

Here we have also used the fact that, since we are dealing with a fluid,

$$
\lim _{t \rightarrow \infty} G(t)=0 \text {. }
$$

In flows in which the deformations are not necessarily small, but the norm $\left\|C_{I}(\tau)-1\right\|$ is still small, for instance since the deformations are sufficiently slow, the stress response can be approximated by the first term of (2.19). Here we are in the domain of what is called 'finite viscoelasticity', which means that the equations are linear in the strain measure, but the strain measure itself is geometrically nonlinear. In this case the isotropic term is often omitted (which is allowed since the fluid is incompressible) and we obtain

$$
T_{\mathrm{E}}=\int_{-\infty}^{t} \mu(t-\tau) C_{r}(\tau) \mathrm{d} \tau
$$

It is important to note that the strain measure in this expression is by no means unique. In fact, instead of (2.26) we could have taken any equation $\boldsymbol{T}_{\mathrm{E}}=\mathscr{F}_{\tau=-\infty}^{\prime}\{\boldsymbol{M}\}$ as a starting point in which $\boldsymbol{M}$ is an isotropic function of $\boldsymbol{C}(\tau)$ and accordingly a different integral expansion would have been obtained. The total value $T_{\mathrm{E}}$ of all such expansions will be the same, but if one considers just a finite number of terms the choice of the strain measure becomes significant. Of the infinite number of alternatives of equation (2.26) we mention only the one based upon the Finger strain tensor:

$$
T_{\mathrm{E}}=\int_{-\infty}^{t} \mu(t-\tau) C_{t}^{-1}(\tau) \mathrm{d} \tau
$$

In $\S \S 4.2$ and 5.2 it will be shown that constitutive equations of this type also follow from particular types of molecular models.

Next we consider the class of slow flows. The qualification 'slow' can be given a precise meaning by the concept of retardation of a flow history. Coleman and Noll (1960) have shown that the functional (2.17) based upon the retarded motion can be approximated by a polynomial of Rivlin-Ericksen tensors (2.11). In this way one obtains a series of approximations

$$
T_{\mathrm{E}}=T_{1}+T_{2}+T_{3}+\ldots
$$

where

$$
\begin{aligned}
& \boldsymbol{T}_{1}=\eta \boldsymbol{A}_{1} \\
& \boldsymbol{T}_{2}=\alpha_{1} \boldsymbol{A}_{2}+\alpha_{2} \boldsymbol{A}_{1}^{2} \\
& \boldsymbol{T}_{3}=\beta_{1} \boldsymbol{A}_{3}+\beta_{2}\left(\boldsymbol{A}_{1} \cdot \boldsymbol{A}_{2}+\boldsymbol{A}_{2} \cdot \boldsymbol{A}_{1}\right)+\beta_{3}\left(\operatorname{tr} \boldsymbol{A}_{2}\right) \boldsymbol{A}_{1} .
\end{aligned}
$$

If only the term with $T_{1}$ is retained we obtain the constitutive equation of a Newtonian fluid. If the terms up to $T_{2}$ are retained one often speaks of a second-order fluid and so on. If the integral expansion (2.19) is valid, relations between the constants in (2.29) and the memory functions in (2.19) may be derived. 
A third property of the flow which may enable a simplification of the general constitutive equation in particular cases is the flow geometry. This geometry may be so simple that the functional (2.17) of a strain history reduces to a function of some flow characteristics. We first illustrate this for the steady simple shear flow. In this flow the velocity $v$ is in the $x$ direction with a gradient in the $y$ direction, so $v=v(y) i$, here $i$ is a unit vector in the $x$ direction. The velocity gradient tensor becomes

$$
L=\dot{\gamma} N
$$

in which $\dot{\gamma}$ is a constant, the rate of shear, and $\boldsymbol{N}=i j$ is a dyadic, based upon the unit vectors $i$ and $j$ in the $x$ and $y$ directions respectively. The relative deformation gradient and the relative Cauchy tensor become

$$
F_{t}(\tau)=\mathbf{1}+(\tau-t) \dot{\gamma} \boldsymbol{N}
$$

and

$$
C_{t}(\tau)=\mathbf{1}+(\tau-t) \dot{\gamma}\left(\boldsymbol{N}+\mathbf{N}^{\mathrm{T}}\right)+(\tau-t)^{2} \dot{\gamma}^{2} \boldsymbol{N}^{\mathrm{T}} \cdot \mathbf{N}
$$

The tensor $C_{t}(\tau)$ is fully determined by two constants: the scalar $\dot{\gamma}$ and the tensor $N$. This means that if the strain history in the function (2.17) in this type of flow is also fully determined by these two quantities. So the extra-stress response becomes

$$
T_{\mathrm{E}}=\underset{\tau=-\infty}{\mathscr{F}}\left\{C_{t}(\tau)\right\}=f(\dot{\gamma}, N)
$$

in which $f$ is a function. One might say that the flow history leaves so little to remember that the functional $\mathscr{F}$ reduces to the function $f$. Next the isotropy (2.18) of the constitutive equation is used. This implies also that the function $f$ is isotropic, and by making use of representation theorems of isotropic tensor functions (Spencer and Rivlin 1959, 1960) the following result is obtained

$$
\boldsymbol{T}_{\mathrm{E}}=\tau(\dot{\gamma})\left(\boldsymbol{N}+\boldsymbol{N}^{\mathrm{T}}\right)+\sigma_{\mathrm{i}}(\dot{\gamma}) \boldsymbol{N} \cdot \boldsymbol{N}^{\mathrm{T}}+\sigma_{2}(\dot{\gamma}) \boldsymbol{N}^{\mathrm{T}} \cdot \boldsymbol{N}
$$

The relatively simple shape of this expression is a consequence of the property $\boldsymbol{N}^{2}=\mathbf{0}$ of the tensor $\boldsymbol{N}$. For arbitary tensors $\boldsymbol{N}$ on the basis of isotropy arguments alone, instead of (2.34), a far more complicated result would have been obtained.

From (2.34) we see that the material behaviour is completely specified by just three functions: $\tau(\dot{\gamma}), \sigma_{1}(\dot{\gamma})$ and $\sigma_{2}(\dot{\gamma})$. Such functions are called viscometric functions. Conventionally, not this set, but the following viscometric functions and coefficients are used.

(1) The shear stress function $\tau(\dot{\gamma})$ and the viscosity $\eta(\dot{\gamma})$ :

$$
\tau(\dot{\gamma})=\eta(\dot{\gamma}) \dot{\gamma}=T_{E}\langle x y\rangle .
$$

(2) The first normal stress function $N_{1}(\dot{\gamma})$ and coefficient $\psi_{1}(\dot{\gamma})$

$$
N_{\mathrm{I}}(\dot{\gamma})=\psi_{1}(\dot{\gamma}) \dot{\gamma}^{2}=T_{\mathrm{E}}\langle x x\rangle-T_{\mathrm{E}}\langle y y\rangle \text {. }
$$

(3) The second normal stress function $N_{2}(\dot{\gamma})$ and coefficient $\psi_{2}(\dot{\gamma})$

$$
N_{2}(\dot{\gamma})=\psi_{2}(\dot{\gamma}) \dot{\gamma}^{2}=T_{\mathrm{E}}\langle y y\rangle-T_{\mathrm{E}}\langle z z\rangle \text {. }
$$

As a next special class of flows we now consider flows with a constant (in the material sense) velocity gradient:

$$
\dot{L}=0(-\infty \leq t \leq \infty) \quad L=\text { constant }
$$


The differential equation, with the given initial condition,

$$
\frac{\mathrm{d}}{\mathrm{d} \tau} F_{t}(\tau)=L \cdot F_{t}(\tau) \quad F_{t}(t)=1
$$

then has the unique solution

$$
F_{t}(\tau)=\exp (\tau-t) L
$$

In this sense, motions with a constant velocity gradient have an exponential flow history. In the case that $L=\dot{\gamma} \boldsymbol{N}$ and $\boldsymbol{N}^{2}=\mathbf{0}$ the series expansion of (2.40) reduces to (2.31) and the steady shear flow is reobtained. The class of flows with $L^{2} \neq 0$ but $\boldsymbol{L}^{3}=0$ contains the subclass of doubly superimposed shear flows. The class with $L^{n} \neq 0$ for all $n=1,2,3, \ldots$ contains some interesting subclasses: triply superimposed shear flows, steady pure shear flows, steady simple extensions and the Maxwell orthogonal rheometer flows (Maxwell and Chartaff 1965, Huilgol 1969). Here we consider in more detail just the extensional flows. In that case $L=D$ is symmetric and in an appropriate orthogonal basis the tensors $D$ and $F_{f}(\tau)$ have the following matrix representations:

$\boldsymbol{D}=\left(\begin{array}{ccc}\dot{\varepsilon}_{1} & 0 & 0 \\ 0 & \dot{\varepsilon}_{2} & 0 \\ 0 & 0 & \dot{\varepsilon}_{3}\end{array}\right) \quad \boldsymbol{F}_{f}(\tau)=\left(\begin{array}{ccc}\exp (\tau-t) \dot{\varepsilon}_{1} & 0 & 0 \\ 0 & \exp (\tau-t) \dot{\varepsilon}_{2} & 0 \\ 0 & 0 & \exp (\tau-t) \dot{\varepsilon}_{3}\end{array}\right)$

Here $\dot{\varepsilon}_{i}(i=1,2,3)$ are constants, the so-called elongation rates. In incompressible fluids $\operatorname{tr} \boldsymbol{D}=0$, and therefore $\dot{\varepsilon}_{1}+\dot{\varepsilon}_{2}+\dot{\varepsilon}_{3}=0$. The most important special case is the uniaxial extension: $\dot{\varepsilon}_{1}=-\frac{1}{2} \dot{\varepsilon}, \dot{\varepsilon}_{2}=-\frac{1}{2} \dot{\varepsilon}, \dot{\varepsilon}_{3}=\dot{\varepsilon}$.

By arguments similar to the ones used in (2.33) the stress response in flows with a constant velocity gradient may be shown to be

$$
T_{\mathrm{E}}=f(L)
$$

in which $f$ is an isotropic function. In extensional flows we have

$$
T_{\mathrm{E}}=f(D)=\phi_{0} 1+\phi_{1} D+\phi_{2} D^{2}
$$

in which the scalars $\phi_{i}$ are functions of the principal invariants of the tensor $\boldsymbol{D}$. In the case of a uniaxial extension the stress response is usually specified by the elongational viscosity $\eta_{\mathrm{E}}$, defined as

$$
\eta_{E}(\dot{\varepsilon})=\frac{T_{E}\langle x x\rangle-T_{E}\langle y y\rangle}{\dot{\varepsilon}} .
$$

So far we have considered only plane flows with straight flow lines. It is, however, possible to generalise the simple shear flow to the more general class of viscometric flows (Coleman 1962) and the flows with a constant velocity gradient to the class of motions with a constant stretch history ( $\mathrm{MCSH}$ ) (Noll 1962). A full discussion of those flows can be found in many textbooks (e.g. Zahorski 1982). Here we will mention only some of the basic motions. The viscometric flows are a special case of the MCSH, therefore we will first consider the latter.

According to the definition of a MCSH and a theorem of Noll (1962), in such a motion the relative deformation gradient is given by

$$
F_{t}(\tau)=Q^{\top}(\tau) \cdot \exp (\tau-t) M_{0} \cdot Q(t)
$$


in which the tensor $\boldsymbol{Q}$ is orthogonal and the tensor $\boldsymbol{M}_{0}$ is constant. From the transformation rules for a change of frame (see Appendix 1) it follows that a ${ }^{*}$-frame exists in which $F_{i}^{*}(\tau)$ has the form

$$
F_{i}^{*}(\tau)=\exp (\tau-t) \boldsymbol{M}_{0} .
$$

So, for a moving observer in the *-frame the flow is of the type (2.40) with a constant velocity gradient $\boldsymbol{L}^{*}=\boldsymbol{M}_{0}$. Since, by the principle of material frame indifference (see $\S 2.2$ ), the stress response is known in any frame if it is known in a particular one, we first consider the stress response in the ${ }^{*}$-frame. Similar to $(2.42)$ we have

$$
T_{\mathrm{E}}^{*}=\underset{\tau=-\infty}{t}\left\{C_{r}^{*}(\tau)\right\}=G\left(M_{0}\right)
$$

From the principle of material frame indifference it follows that

$$
T_{\mathrm{E}}=\underset{\tau=-\infty}{\mathscr{P}}\left\{C_{\mathrm{r}}(\tau)\right\}
$$

Finally, noting that $C_{r}(\tau)$ is the same function of $\boldsymbol{M}=Q^{\top}(t) \cdot \boldsymbol{M}_{0} \cdot \boldsymbol{Q}(t)$ as $C_{t}^{*}(\tau)$ is of $\boldsymbol{M}_{0}$, which may be proved from (2.44) and (2.45), we see that

$$
T_{E}(t)=G(M(t))
$$

in which $G$ is the same function as in equation (2.47). This result, in principle, determines the stress response in arbitrary MCsHs and in viscometric flows. The time dependence in (2.49) is a trivial one, since it is just a consequence of the change of frame. In the ${ }^{*}$-frame, the stress response and the tensor $\boldsymbol{M}_{0}$ as well, are constants.

\subsection{Special constitutive equations}

In arbitrary flows the constitutive equations obtained from simple fluid theory are too general for practical applications. For specific classes of materials, however, from empirical as well as from microscopical considerations, special constitutive equations may be obtained. In this section some examples of such equations for polymeric systems will be discussed.

First we have the so-called generalised Newtonian fluids. These are of the type $\boldsymbol{T}_{\mathrm{E}}=2 \eta \boldsymbol{D}$ in which $\eta$ is a function of $\eta=\eta\left(I I_{\boldsymbol{D}}\right)$ of the second invariant of the rate of strain tensor. In steady shear flows then $\eta=\eta(\dot{\gamma})$, a shear-rate-dependent viscosity. For this function many empiricisms exist. For high shear rates the simple 'power-law'

$$
\eta=m \dot{\gamma}^{n-1}
$$

with the parameters $m$ and $n$, usually gives a good description. If the law shear-rate region is included more elaborate functions like the Carreau model

$$
\frac{\eta-\eta_{\infty}}{\eta_{0}-\eta_{\infty}}=\left[1+(\lambda \dot{y})^{2}\right]^{(n-1) / 2}
$$

(Carreau 1968) may be used.

A disadvantage of the generalised Newtonian fluids is that they do not predict normal stress differences in steady shear flow and no relaxation effects in transient flows. A simple generalisation showing both non-Newtonian viscosity and normal stress effects is the so-called Reiner-Rivlin fluid. Here one assumes $T_{E}=f(D)$ where $f$ is an isotropic function, so (omitting isotropic terms)

$$
T_{\mathrm{E}}=\phi_{1} D+\phi_{2} D^{2}
$$


in which $\phi_{1}=\phi_{1}\left(I I_{D}, I I I_{D}\right)$. The equality of normal stress functions, predicted by this model, however, is at variance with experimental data. A similar type of constitutive equation is the CEF equation (Crimale et al 1958)

$$
\boldsymbol{T}_{\mathrm{E}}=\eta \boldsymbol{A}_{1}-\frac{1}{2} \Psi_{1} \boldsymbol{A}_{2}+\left(\Psi_{1}+\Psi_{2}\right) \boldsymbol{A}_{1}^{2}
$$

in which $\eta, \Psi_{1}$ and $\Psi_{2}$ are the viscometric functions $(2.35-7)$ in which for arbitrary flow the shear rate $\dot{\gamma}$ is changed into an invariant of the rate of strain tensor. For viscometric flows this equation is equivalent to the general equation (2.15). For slow flows it may be considered as a second-order fluid in the retarded motion expansion (2.29). For arbitrary flows, however, it is an empirical constitutive equation.

Relaxation phenomena may be described by constitutive equations which are differential equations. The simplest cases are the so-called generalised Maxwell models. These are of the form

$$
T_{\mathrm{E}}+\lambda \frac{\delta}{\delta t} T_{\mathrm{E}}=2 \eta D
$$

in which $\lambda$ and $\eta$ are constant parameters and $\delta / \delta t$ is a convective time derivative (see Appendix 2), so

$$
\frac{\delta}{\delta t} T_{\mathrm{E}}=\stackrel{\mathrm{̆}}{\mathrm{T}}_{\mathrm{E}}, \stackrel{\Delta}{\mathrm{T}}_{\mathrm{E}}, \stackrel{\nabla}{\mathrm{T}}_{\mathrm{E}}, \ldots
$$

In any of those cases a different type of model is obtained (the 'corotational', 'lower convected', 'upper convected'... and so on). At the level of continuum mechanics there is no fundamental reason for preferring any type of convected derivative. In practice the choice is based upon empirical and microscopic considerations. The corotational derivative is related to a frame of reference rotating with the vorticity of the macroscopic flow (Goddard and Miller 1966, Goddard 1967, Huilgol 1978). This turns out to be relevant in dilute solutions of rigid molecules (Abded-Khalic et al 1974, Bird et al 1974). Later on in this review it will be shown that the upper convected derivative is consistent with particular microscopical models, based upon deformable particles. A slight generalisation of these models, obtained by introducing some kind of slip of the microstructure, leads to the Gordon and Schowalter convective derivative $\widetilde{T}_{\mathrm{E}}$ (Gordon and Schowalter 1972, Phan-Thien and Tanner 1977, Johnson and Segalman 1977).

Models in which time derivatives of the rate of strain tensor also occur are of the Jeffrey type

$$
T_{\mathrm{E}}+\lambda_{1} \frac{\delta}{\delta t} T_{\mathrm{E}}=2 \eta\left(D+\lambda_{2} \frac{\delta}{\delta t} D\right)
$$

Again $\delta / \delta t$ may be taken to be one of the various convected time derivatives. In the case that the lower convected derivative is chosen the model is also known as the Oldroyd A-fluid and in the case of the upper convected derivative one speaks of the Oldroyd B-fluid. If the general derivatives (A2.18) are used the model contains, besides the three parameters $\lambda_{1}, \lambda_{2}$ and $\eta$, five additional parameters in the derivatives (not six, since one term in the derivative of $D$ drops out because tr $D=0$ as a result of the assumed incompressibility). This is known as the Oldroyd eight-constant model (Oldroyd 1958, 1961). 
So far we have considered differential equations with constant coefficients (quasilinear models). Often, however, in order to improve the rheological predictions particular nonlinearities are introduced in the model.

By taking the relaxation time $\lambda$ in the upper convected Maxwell model to be a function of the second invariant of the rate of strain tensor and the viscosity equal to $\eta=G \lambda$ where $G$ is a constant, the White-Metzner (1963) model is obtained:

$$
\boldsymbol{T}_{\mathrm{E}}+\lambda\left(I I_{D}\right) \stackrel{\nabla}{\boldsymbol{T}_{\mathrm{E}}}=2 G \lambda\left(I I_{D}\right) D .
$$

This model has the advantage of being relatively simple and yet giving reasonable predictions for the shear-rate-dependent viscosity and first normal stress functions.

Nonlinearities in the Maxwell model may also be introduced by using stressdependent coefficients or by adding nonlinear stress-dependent terms. An example of the first category is the Phan-Thien-Tanner (1977) model (see also § 5.3)

$$
\sigma\left(\operatorname{tr} T_{\mathrm{E}}\right) T_{\mathrm{E}}+\lambda \overline{\bar{T}}_{\mathrm{E}}=2 \eta(1-\zeta) D
$$

in which also the Gordon-Schowalter convected derivative (A2.16) has been used. An example of the second kind is a model proposed by Giesekus (1982) (see also $\$ 7.3$ )

$$
T_{\mathrm{E}}+\lambda \bar{T}_{\mathrm{E}}+a T_{\mathrm{E}}^{2}=2 \eta D
$$

Besides the special constitutive equations of the differential type one often considers equations of the integral type. The starting point here is the integral expansion (2.19). The convergence of this expansion may be shown (Bird et al 1975) to be strongly dependent upon the strain measure, used in the expansion. In (2.19) the expansion was based upon the tensor $C_{t}(\tau)$. In practice one usually takes only the single integral term and optimises the choice of the strain measure and the memory function. As noted already, the Finger tensor $C_{t}^{-1}(\tau)$ often turns out to be a reasonable choice, so one starting point will be equation (2.27).

Although this model, known as Lodge's rubber-like liquid (Lodge 1964) has some attractive features, it is not capable of describing-even qualitatively-many of the rheological properties of polymeric liquids. (See, for instance, Tanner (1983) for a discussion of the usefulness of the Lodge model and some related models in various types of flow.)

In order to improve the Lodge model, one may consider the case of norlinear memory functions. In that case we have

$$
\mu=\mu(t-\tau,\{I\})
$$

where $\{I\}$ stands for a set of invariants of the rate of strain tensor $D(\tau)$, the stress tensor $T_{\mathrm{E}}(\tau)$ or of the Finger tensor $C_{t}^{-1}(\tau)$. An example of the first type is the model of Bird and Carreau (1968). An example of the second category is the model of Phan-Thien and Tanner (1977). A disadvantage of this type of model is that the constitutive equation is no longer explicit, but instead an integral equation in stress. In the third case we have

$$
\mu=\mu(t-\tau, I, I I)
$$

in which $I$ and $I I$ are the two first principal invariants of the Finger tensor: $I=\operatorname{tr} C_{t}^{-1}(\tau)$; $I I=\operatorname{tr} C_{t}(\tau)$. The corresponding constitutive equation is a special case of the RivlinSawyers equation

$$
T_{\mathrm{E}}=\int_{-\infty}^{t}\left[\psi_{1}(t-\tau, I, I I) C_{t}(\tau)+\psi_{2}(t-\tau, I, I I) C_{t}^{-1}(\tau)\right] \mathrm{d} \tau
$$


which may be proved (Rivlin and Sawyers 1971) to be the most general constitutive equation of a fluid under the assumption that the effects on the stress at time $t$ of the deformations at different past times $t^{\prime}$ are independent of each other. In the case that a potential $W(t-\tau, I, I I)$ exists, such that

$$
\psi_{1}=\frac{\partial W}{\partial I} \quad \psi_{2}=\frac{\partial W}{\partial I I}
$$

(2.62) is the famous K-BKZ equation (Kaye 1962, Bernstein et al 1963). The functions $\psi_{1}$ in (2.62) may contain a common factor $\mu(t-\tau)$. In that case the integrand becomes separable, i.e. a product of a time-dependent and a strain-dependent function:

$$
T_{\mathrm{E}}=\int_{-\infty}^{t} \mu(t-\tau)\left[\phi_{1}(I, I I) C_{t}(\tau)+\phi_{2}(I, I I) C_{t}^{-1}(\tau)\right] \mathrm{d} \tau .
$$

A well known special case is the model of Wagner (1979) in which $\phi_{1}=0$ and $\phi_{2}=H(I, I I)$ the so-called damping function (see also $\S 5.3$ ). The general case (2.64) belongs to the class of equations of the type

$$
\boldsymbol{T}_{\mathrm{E}}=\int_{-\infty}^{t} \mu(t-\tau) \boldsymbol{M}_{t}(\tau) \mathrm{d} \tau
$$

in which $\boldsymbol{M}_{t}(\tau)$ is a generalised strain measure. Equation (2.64) is obtained if $\boldsymbol{M}_{t}(\tau)$ is supposed to be an isotropic function of the tensor $C_{t}(\tau)$.

A different class of generalised strain measures is obtained by defining $\boldsymbol{M}_{t}(\tau)$ by the differential equation

$$
\frac{\mathrm{d}}{\mathrm{d} \tau} \boldsymbol{M}_{i}(\tau)=2 \Gamma(t, \tau): D(\tau)
$$

in which $\Gamma(t, \tau)$ is an entrainment law as was used in the definition of convective derivatives (see Appendix 2). In the lower convected case we have

$$
\frac{\mathrm{d}}{\mathrm{d} \tau} \boldsymbol{M}_{t}(\tau)=2 \boldsymbol{F}_{t}^{\mathrm{T}}(\tau) \cdot \boldsymbol{D}(\tau) \cdot \boldsymbol{F}_{t}(\tau)
$$

so

$$
\boldsymbol{M}_{t}(\tau)=1+2 \int_{t}^{\tau} \boldsymbol{F}_{t}^{\mathrm{T}}(\tau) \cdot \boldsymbol{D}(\tau) \cdot \boldsymbol{F}_{i}(\tau) \mathrm{d} \tau=\boldsymbol{C}_{t}(\tau)
$$

and in the upper convected case

$$
\frac{\mathrm{d}}{\mathrm{d} \tau} \boldsymbol{M}_{\mathrm{t}}(\tau)=-2 \boldsymbol{F}_{\mathrm{t}}^{-1}(\tau) \cdot \boldsymbol{D}(\tau) \cdot \boldsymbol{F}_{\mathrm{i}}^{-\mathrm{T}}(\tau)
$$

so

$$
M_{t}(\tau)=1-2 \int_{1}^{\tau} F_{t}^{-1}(\tau) \cdot D(\tau) \cdot F_{t}^{-\tau}(\tau)=C_{t}^{-1}(\tau) .
$$

In these cases we reobtain constitutive equations of the type (2.65). For the co-rotational 
case, however, the strain measure becomes (Goddard 1967, 1979)

$$
\boldsymbol{M}_{t}(\tau)=1+2 \int_{t}^{\tau} \overline{\boldsymbol{R}}_{t}^{\mathrm{T}}(\tau) \cdot \boldsymbol{D}(\tau) \cdot \overline{\boldsymbol{R}}_{t}(\tau) \mathrm{d} \tau
$$

in which $\overline{\boldsymbol{R}}_{t}$ is the mean rotation tensor used in (A2.13). In this case $\boldsymbol{M}_{t}(\boldsymbol{\tau})$ is not expressible as a function of $\boldsymbol{C}_{t}(\tau)$.

If the entrainment law is based upon the Gordon-Schowalter convected derivative (see Appendix 2) the corresponding strain tensor becomes

$$
\boldsymbol{M}_{t}(\tau)=1+2 \int_{t}^{\tau} \tilde{\boldsymbol{F}}_{t}^{-1}(\tau) \cdot \boldsymbol{D}(\tau) \cdot \tilde{\boldsymbol{F}}_{t}^{-\mathrm{T}}(\tau) \mathrm{d} \tau=\frac{1}{1-\zeta} \tilde{\boldsymbol{C}}_{t}^{-1}(\tau)
$$

in which $\tilde{\boldsymbol{C}}_{t}^{-1}(\tau)$ is the Finger tensor, based upon the effective deformation gradient $\tilde{F}_{t}(\tau)$. Also in this case, the strain tensor $\boldsymbol{M}_{t}(\tau)$ is not expressible as a function of $C_{t}(\tau)$. For a further discussion on this type of 'noholonomic' strain we refer to a paper by Goddard (1979).

Partial integration of (2.65) and substitution of (2.66) gives

$$
T_{\mathrm{E}}=2 \int_{-\infty}^{t} \nu(t-\tau) \Gamma(t, \tau): D(\tau) \mathrm{d} \tau
$$

in which

$$
\nu(t)=\int_{t}^{\infty} \mu(s) \mathrm{d} s
$$

By using the different entrainment laws, alternative forms of the constitutive equations corresponding to the expressions (2.68), (2.70), (2.71) and (2.72) of $\boldsymbol{M}_{t}(\tau)$ are obtained.

\section{Elements of modelling}

\subsection{Introduction}

In continuum mechanics a material is represented by a particular model: the continuum. In $\S 2$ we have seen that this model provides a very useful framework for a phenomenological treatment of constitutive equations and special types of flow. The continuum model, however, is not able to represent any specific information about the microstructure of the system. Instead, one has to deal with a number of unspecified model parameters.

In order to relate these parameters of a system to aspects of its microstructure one needs microscopical models in which the real microstructure is represented by an artificial structure, similar to the original one, but of a more or less simplified shape. Actually there are many ways of representing a given system by a microscopical model. A polymer melt, for instance, may be represented by a coil of entangled chains (Bueche 1956), by a bead-rod chain in a medium with anisotropic viscous friction (Curtiss and Bird 1981), by a Gaussian chain confined in a tube (de Gennes 1971) and so on. Which type of modelling is useful in a given situation depends upon the purpose. In the context of processing, for instance, one usually needs less detailed models than in the development of new materials with specific mechanical properties.

Three important elements of any micro-rheological model are: the structure by which the system is represented, the evolution equations, describing the changes of 
structure in time, and finally the averaging procedure, connecting the macroscopic stress tensor with microscopic stresses and forces. These items will be discussed in $\S \S 3.2$ and 3.3 .

\subsection{Structure}

A polymeric chain may be represented by model representations at various levels of description. A rather refined description is a chain of coupled rods with fixed bond length and angles in which the rotation around the rods may be taken as hindered (Flory 1969) or free (Kirkwood 1967). A more crude representation of the polymeric chain is a freely jointed chain or 'Kramers chain' of $N$ beads, connected by $N-1$ rods (Kramers 1944). The beads do not represent atoms of the polymer chain but merely points in the model in which the frictional forces are acting and the rods have a length in accordance with the requirement of free jointedness. It is also possible to represent larger parts of the molecule by beads, connected by elastic springs, instead of rods. In that case one obtains the bead-spring model, well known from the theory of Rouse (1953) and Zimm (1956) for the viscoelastic behaviour of dilute polymer solutions. A very crude description of the molecule, which, however, is often useful because of its simplicity, is an elastic dumbbell, consisting of two beads, connected by one spring. Rod-like molecules may be modelled by a rigid dumbbell, consisting of two beads, connected by a rod. Some bead-rod-spring models will be discussed in $\S 4.2$; for an extensive treatment we refer to the textbook of Bird et al (1987b).

So far we have only considered the representation of one polymer molecule by a model. This is often sufficient in dilute systems. In concentrated systems, however, the model should also represent the intermolecular interactions. A simple but rather successful approach is the transient-network model (Lodge 1956, Yamamoto 1956) in which the strongly interacting polymer molecules are represented by a temporary network of segments which are created and annihilated with prescribed probabilities. This model will be discussed in $\S 5$.

A less artificial model for a concentrated polymeric system than a temporary network is a set of 'entangled' molecules. In such a system the molecules interact with each other by entanglements in which some kind of slip occurs if the molecules are in relative motion. For a review on this type of theory we refer to Graessley (1974). Although rather successful in the prediction of some rheological functions, the existing entanglement theories are not entirely satisfactory because of the many ad hoc assumptions that are involved. A rigorous treatment of a system of entangled polymers, however, turns out to be an extremely difficult mathematical problem.

A rather successful class of models for concentrated systems are the reptation theories. These are mean field theories, in which one central molecule is embedded in some kind of special environment, representing the topological constraints caused by the neighbouring molecules. Two important cases are the tube model (de Gennes 1971, Doi and Edwards 1978, 1979) in which the molecule is confined in a tube, and the model of Curtiss and Bird (1981) in which the molecule is embedded in a viscous fluid with hydrodynamic forces determined by an anisotropic Stokes' law. As a result, in both cases, the motion of the central molecule will be one along its own contour, in some average sense. This 'snake-like' motion is called reptation. Reptation models will be discussed in $\S 6$.

Having specified the model representation of a molecule, or a whole set of entangled molecules, we next have to specify the state of the system. The most detailed description 
of this state would be the specification of all coordinates and momenta associated with the total system (in the chosen model representation). Such a detailed description of state may be useful for computer simulations but not for further analytical considerations. The next level of description is the use of probability distributions. In the most fundamental one uses a probability density $\Phi(P, Q, t)$ of generalised momenta and coordinates. An extensive discussion of this so-called phase-space kinetic approach can be found in the textbook by Bird et al (1987b). In many cases, however, it is sufficient to assume equilibrium in momentum space and consider only the configurational distribution function $\Psi(q, t)$ in order to specify the state of a system. In some cases the microstate of a system may be sufficiently well characterised by a set of structure tensors $\{\boldsymbol{S}\}$. These may be moments of the distribution function $\Psi$ but also a set of variables that are introduced ad hoc. In the latter case we are dealing with the configurational tensor models, to be discussed in $\S 7$.

At any level of description of state one needs equations determining the evolution of state. At the lowest level of description these equations are the mechanical equations of motion and at the phase-space kinetic level this is the classical Liouville equation for the distribution function $\Phi(P, Q, t)$. At the next level of description specific model assumptions may be introduced in the evolution equations. In the case of bead-rodspring models the treatment is usually based upon the theory of Brownian motion (see for instance the book by Wax (1954) in which some of the classical papers on this subject are collected) and a Smoluchowski equation of the type

$$
\frac{\partial \Psi(Q, t)}{\partial t}=-\Gamma(\Psi(Q, t))
$$

in which $\Gamma$ is a linear differential operator depending on $Q$ and $t$ (see also Doi and Edwards (1986) for further details). The specific form of the operator $\Gamma$ depends upon the details of the model. In general one may distinguish convective, diffusive and kinetic contributions. In the bead-rod-spring models, which will be discussed in $\S 4$, the operator $\Gamma$ is of a purely convective and diffusive nature. This follows from a consideration of the balance of hydrodynamic, Brownian and interaction forces. With Brownian forces we mean the force field in $Q$ space, usually given by an expression like

$$
f^{\mathrm{B}}=-k T \frac{\partial}{\partial Q} \ln \Psi(Q, t)
$$

which may be considered as the force field associated with the diffusive part of the motion in $\Phi$ space.

In transient network models, to be discussed in $\S 5$, the evolution equation is of the form

$$
\frac{\partial \Psi(Q, t)}{\partial t}=-\Gamma[\Psi(Q, t)]+\Lambda(Q, t)
$$

in which the operator $\Gamma$ is of a purely convective nature and a source term $\Lambda$ is added in order to describe the processes of creation and annihilation of structural elements.

Reptation models, to be discussed in $\$ 6$, may also be based upon a differential equation of the type (3.3). In that case the operator $\Gamma$ is convective as well as diffusive.

At the level of description where the state of a system is described by a set of structure tensors $S$ the evolution equations usually are of the type

$$
\dot{S}=f(S, L)
$$


in which $\boldsymbol{L}$ is the macroscopic velocity gradient (2.8). Such equations may be derived from the underlying evolution equations for $\psi(Q, t)$ or introduced as ad hoc assumptions, at the level of the structure tensors. A discussion of rheological models based upon this level of description will be given in $\$ 7$.

\subsection{The average stress tensor}

In order to predict macroscopic rheological properties, a micro-rheological model should contain an expression which relates the macroscopic stress tensor to the variables by which the microstructure is described. So, depending upon the level of description of the microstructure there will be various expressions of the stress tensor of a given system. There are also several procedures for deriving these expressions. Most of them are based upon some kind of averaging. In disperse systems one often employs the method of volume averaging (Batchelor 1970) which may be useful in other kinds of systems too (Jongschaap and Doeksen 1983). An elementary, but very useful procedure, due to Kramers (1944), is based upon the averaging of all forces, transmitted through a plane in the medium. A more fundamental approach, due to Irving and Kirkwood (1950), is based upon a statistical mechanical derivation of the macroscopical transport equations. This method has been investigated extensively by Curtiss, Bird and Hassager (see chapter 17 of the textbook by Bird et al (1987b)).

An expression for the average stress tensor may also be derived from a virtual work principle. This approach is based upon the macroscopic expression

$$
W=V T: L
$$

for the stress power in a volume $V$ of a continuum. By equating this to a microscopic expression for $W$ an expression for $\boldsymbol{T}$ may be derived. This method, applied by Batchelor (1977) to disperse systems and by Doi (1983) to polymeric systems, will also be taken as a starting point in this review (closely following a paper by Jongschaap (1987)). An advantage is that it may be applied consistently to various levels of description of the microstructure (see $\$ 3.1$ ).

As a simple illustration we consider a set $\alpha$ of particles, which may be a subset of a larger system, consisting of other kinds of particles. In a homogeneous deformation in which the particles of the subset $\alpha$ are forced to move affinely with the macroscopic deformation the power supplied to the subset becomes

$$
W_{\alpha}=\sum_{i \in \alpha} f_{i}^{\alpha^{\prime}} \cdot \boldsymbol{L} \cdot \boldsymbol{r}_{i}=\sum_{i \in \alpha} f_{i}^{\alpha^{\prime}} \boldsymbol{r}_{i}: L
$$

Here, the force $f_{i}^{\alpha^{\prime}}$ is the total force acting upon the particle $i$ due to influences from outside the subset $\alpha$.

Instead of the velocity gradient $L$ and the power $W_{\alpha}$, one could also consider virtual displacements and the associated virtual work $\delta W_{\alpha}$. In the following we will keep the notations of (3.5) and (3.6) but still consider the processes as virtual. So, in these considerations the time is treated as an arbitrary parameter specifying the virtual displacements and the tensor $\boldsymbol{L}$ as a virtual displacement gradient.

By identifying the expressions (3.5) and (3.6) an expression for the contribution $T_{\alpha}$ of the subset $\alpha$ to the stress tensor is obtained:

$$
T_{\alpha}=\frac{1}{V} \sum_{i \in \alpha} f_{i}^{\alpha^{\prime}} \boldsymbol{r}_{i}
$$


In the case of a dilute polymer solution in which the molecules are modelled by bead-rod-spring systems this expression may be applied to a subset $p$ consisting of a large number of molecules. Then the forces $f_{i}^{p^{\prime}}$ are the hydrodynamic forces $f_{i}^{\mathrm{H}}$ on the beads and by changing the summation into an ensemble average we obtain

$$
T_{p}=n \sum_{i}\left\langle f_{i}^{\mathrm{H}} \boldsymbol{r}_{i}\right\rangle
$$

in which $n=N / V$ is the number density of molecules and the summation is restricted to one molecule. This expression may also be derived by the method of volume averaging. In that case, for a particle of an arbitrary shape, the particle contribution becomes

$$
T_{p}=n\left\langle\int_{V_{p}} \boldsymbol{\sigma} \mathrm{d} V\right\rangle
$$

in which $\boldsymbol{\sigma}$ is the stress tensor inside a particle. By transforming the volume integral (3.9) into a surface integral (see Batchelor 1970) one obtains

$$
T_{p}=n\left\langle\int_{\partial V_{p}} \sigma \cdot n r \mathrm{~d} S\right\rangle .
$$

In applying this formula to a bead-rod-spring model the contribution of a bead to the integral becomes $\boldsymbol{f}_{i}^{\mathrm{H}} \boldsymbol{r}_{i}$ and the result (3.8) is reobtained.

The expressions (3.9) and (3.10) are also relevant in the case of reptation models of polymeric liquids (see $\S 6$ ). In that case we consider a polymer molecule modelled as a particle with the shape of a rope. If $e$ is the local tangent vector along its contour and the stress is taken to be $\sigma=\sigma e e$ we obtain from (3.9)

$$
T_{p}=n\left\langle\int_{0}^{L} \text { oee } \mathrm{d} S\right\rangle
$$

in which $L$ is the contour length. An expression of this type was used by Doi and Edwards $(1978,1979)$. If, on the other hand (3.10) is applied to the case of a rope we obtain

$$
T_{p}=n\left\langle\int_{0}^{L} f r \mathrm{~d} S\right\rangle
$$

in which $f$ is the external force, acting upon it. An equation of this type was used by Curtiss and Bird (1981) in their kinetic theory of polymer melts. In their case $f$ was an anisotropic hydrodynamic force.

In the procedure of volume averaging it is evident that the result (3.8) is unaffected by constraints (e.g. rigid rods). The derivation, based upon a virtual work principle, seems, however, not to be valid in that case since constraints may prevent the system from following the virtual deformation used in (3.6). This, however, is not true. The virtual work principle, used to derive (3.8) is still valid in constrained systems. The reason is that the stress in a constrained system is based upon an equilibrium of internal forces. If a system with constraints is changed into one without constraints which is still able to resist the internal forces (for instance by changing a rigid rod into a spring with a modulus, sufficient to keep its length fixed for the given force) the stress will remain unaltered. An expression for the stress tensor is obtained then by considering virtual motions of the unconstrained system. 
The expression (3.8) for the stress tensor may be specified further by introducing an expression for $f^{H}$ or by eliminating $f^{H}$ by using an equilibrium of forces. This will be illustrated now for the case of a dumbbell model. Denoting the end-to-end vector of the dumbbell by $q$ and its centre by $r_{0}$ we obtain from (3.8) $T_{p}=n\left\langle f_{0}^{\mathrm{H}} r_{0}\right\rangle+n\left\langle f^{\mathrm{H}} q\right\rangle$ in which $f_{0}^{\mathrm{H}}=f_{1}^{\mathrm{H}}+f_{2}^{\mathrm{H}}$, the total hydrodynamic force on the centre and $f^{\mathrm{H}}=\frac{1}{2}\left(f_{2}^{\mathrm{H}}-f_{1}^{\mathrm{H}}\right)$, an 'internal' hydrodynamic force. In the first term $f_{0}^{\mathrm{H}}$ is balanced by a Brownian force $f_{0}^{\mathrm{B}}=-k T\left(\partial / \partial \boldsymbol{r}_{0}\right)$ In $\psi$ associated with the spatial diffusion of the whole particle. By partial integration it can be shown that this term becomes $n\left\langle f_{0}^{\mathrm{H}} r_{0}\right\rangle=-n k T 1$. This contribution is of no further interest and will be omitted in the subsequent discussion; so we have

$$
T_{p}=n\left\langle f^{\mathrm{H}} q\right\rangle .
$$

From Stokes' law of hydrodynamic resistance it follows that

$$
f^{\mathrm{H}}=-\frac{1}{2} \zeta(\dot{q}-L \cdot q) \text {. }
$$

If this expression is introduced in (3.13) making use of the symmetry of the stress tensor and the fact that $\mathrm{d} / \mathrm{d} t\langle q q\rangle=\langle\dot{q} \boldsymbol{q}\rangle+\langle\boldsymbol{q} \dot{q}\rangle$ one obtains

$$
T_{p}=-\frac{1}{4} n \zeta \frac{\delta}{\delta t}\langle q q\rangle
$$

in which $\delta / \delta t$ denotes an upper convected derivative (see Appendix 2). This is an example of a Giesekus expression of the stress tensor (Giesekus 1962).

We now introduce the Brownian force (3.2)

$$
f^{\mathrm{B}}=-k T \frac{\partial}{\partial \boldsymbol{q}} \ln \psi
$$

and the connector force

$$
f^{1}=-k T \ln \psi_{0}=\kappa q
$$

in which $\kappa$ is a spring modulus.

Then, the equilibrium of forces becomes

$$
f^{\mathrm{H}}+f^{\mathrm{B}}-f^{1}=\mathbf{0} \text {. }
$$

With this result, the stress tensor expression (3.13) becomes

$$
\boldsymbol{T}_{\boldsymbol{p}}=-n\left\langle\boldsymbol{f}^{\mathrm{B}} \boldsymbol{q}\right\rangle+n\left\langle\boldsymbol{f}^{1} \boldsymbol{q}\right\rangle
$$

or, on substituting (3.16) and (3.17) and integration by parts:

$$
T_{p}=-n k T 1+n \kappa\langle q q\rangle .
$$

This result, the so-called 'Kramers expression' (Kramers 1944) may also be derived by averaging the forces transmitted by the connectors and the momentum transfer through a plane in the fluid (see for example Bird et al 1987b).

If the dumbbell is rigid, a constraint

$$
|q|=q=\text { constant }
$$

is imposed upon the vector $q$. The consequences of this constraint may be expressed conveniently by introducing the projection operator

$$
P=1-e e
$$


with $e=q /|q|$. The force $f^{\mathrm{H}}$ may then be decomposed as

$$
\boldsymbol{f}^{\mathrm{H}}=\boldsymbol{P} \cdot \boldsymbol{f}^{\mathrm{H}}+(\mathbf{1}-\boldsymbol{P}) \cdot \boldsymbol{f}^{\mathrm{H}} \text {. }
$$

The first term, perpendicular to $e$, is balanced by the Brownian force $\boldsymbol{P} \cdot \boldsymbol{f}^{\mathrm{B}}$ as can be seen by multiplying the balance of forces (3.18) by $\boldsymbol{P}$ and noting that $\boldsymbol{P} \cdot \boldsymbol{f}^{\mathrm{I}}=\mathbf{0}$. The second term in (3.23) is balanced by $f^{1}$, which is a constraining force, determined by the external force $f^{\mathrm{H}}$. The expression (3.13) for $T_{p}$ now becomes

$$
T_{p}=-n\left\langle\boldsymbol{P} \cdot f^{\mathrm{B}} \boldsymbol{q}\right\rangle+n\left\langle(1-\boldsymbol{P}) \cdot f^{\mathrm{H}} \boldsymbol{q}\right\rangle .
$$

After substitution the expressions (3.22) for $P$, (3.16) for $f^{B},(3.14)$ for $f^{\mathrm{H}}$ and performing an integration by parts in the first term, the expression for particle contribution to the stress tensor of a dilute solution of dumbbells is obtained:

$$
T_{p}=n k T\langle 3 e e-1\rangle+\frac{1}{2} n \zeta q^{2}\langle e e e e\rangle: D .
$$

So far we have considered dilute solutions of constrained or unconstrained subsystems. We now return to the general case of a subset $\alpha$ of a system of $N$ clusters $(a, b, \ldots)$ of $M$ particles $(i, j, \ldots)$ each. Then for the contribution $T_{\alpha}$ of the subset $\alpha$ to the stress tensor, from (3.7) we obtain

$$
T_{\alpha}=\frac{1}{V} \sum_{i, a} f_{i a}^{\alpha^{\prime}} \boldsymbol{r}_{i a}
$$

where $f_{i a}^{\alpha^{\prime}}$ is the force acting upon particle $i$ in a cluster $a$ from influences outside the subset $\alpha$. We rewrite this expression as

$$
T_{\alpha}=\frac{1}{V} \sum_{l, a} f_{i a}^{\alpha^{\prime}}\left(\boldsymbol{r}_{i a}-\boldsymbol{r}_{a}\right)+\frac{1}{V} \sum_{a} f_{a}^{\alpha^{\prime}} \boldsymbol{r}_{a}
$$

in which

$$
f_{a}^{\alpha^{\prime}}=\sum_{i} f_{i a}^{\alpha^{\prime}}
$$

the total force on the cluster $a$, and

$$
\boldsymbol{r}_{a}=\frac{1}{M} \sum_{i} \boldsymbol{r}_{i a}
$$

the centre of the cluster.

As an example, consider a network consisting of $N$ rod-like segments, connected with each other at their end points. By considering a segment as a subset $\alpha$ consisting of one cluster $a$ and noting that on each segment just two forces $f_{i a}^{\alpha^{\prime}}$ apply, and the total force $\boldsymbol{f}_{a}^{\alpha^{\prime}}$ vanishes, we obtain from (3.27) $\boldsymbol{T}_{\alpha}=(1 / V) \boldsymbol{f}_{\alpha}^{\prime} \boldsymbol{q}_{\alpha}$, in which $\boldsymbol{q}_{\alpha}$ is a segment vector and $f_{\alpha}^{\prime}$ is the external force acting upon the particle at the endpoint of the vector $q_{\alpha}$. (This force is also equal to the force $f_{\alpha}$, transmitted through the segment.) By introducing an appropriate ensemble average the total stress tensor for the network may be written now as

$$
T_{p}=n\langle f q\rangle
$$

in which $f$ and $q$ correspond to $f_{\alpha}^{\prime}$ and $\boldsymbol{q}_{\alpha}^{\prime}$ respectively and $n=N / V$.

As a second application of the expression (3.27) we consider a subset $p$ of macromolecules in a concentrated solution. According to the equilibrium of forces we have

$$
f_{a}^{p^{\prime}}+f_{a}^{p}+f_{a}^{\mathrm{B}}=0
$$


in which $\boldsymbol{f}_{a}^{p}$ is the force on macromolecule $a$ due to interactions with the other macromolecules of the subset $p$ and $f_{a}^{\mathrm{B}}$ the Brownian force on molecule $a$. On using (3.31), the identity

$$
\sum_{\substack{a \\ a \in p}} f_{a}^{p} \boldsymbol{r}_{a}=-\frac{1}{2} \sum_{\substack{a, b \\(a \neq b ; a, b \in p)}} f_{a b} \boldsymbol{r}_{a b}
$$

which may be derived by assuming pair interactions $f_{a b}=-f_{b a}$ and (3.29), we obtain from (3.27)

$$
T_{p}=-\frac{1}{V} \sum_{\substack{a \\ a \in p}} f_{a}^{\mathrm{B}} r_{a}+\frac{1}{V} \sum_{\substack{i, a \\ a \in p}} f_{i a}^{p^{\prime}}\left(\boldsymbol{r}_{i a}-r_{a}\right)+\frac{1}{2 V} \sum_{\substack{a, b \\ a, b \in p}} f_{a b} r_{a b}
$$

or, after changing the summations into appropriate ensemble averages,

$$
T_{p}=T_{p}^{\mathrm{B}}+n\left\langle f_{i}^{\mathrm{H}}\left(\boldsymbol{r}_{i}-\boldsymbol{r}_{0}\right)\right\rangle+\frac{1}{2} n \sum_{a} \overline{f_{0 a} \boldsymbol{r}_{0 a}} .
$$

In this expression, which was first derived by Fixman (1965) in a different way, $T_{p}^{B}$ (usually an isotropic stress) results from the Brownian force term in (3.33) and $f_{i}^{\mathrm{H}}$, the average frictional force on particle $i$ of a cluster, results from averaging the force $f_{i a}^{p^{\prime}}$ for a particular configuration of molecule $a$ with respect to the configurations of all other molecules. The angular brackets in (3.34) denote an average with respect to the internal configurations of a molecule with fixed $\boldsymbol{r}_{0}$ and the overbar in the last term an average for fixed $r_{0 a}$ with respect to the configurations of all other molecules.

For concentrated polymer solutions or melts, an interesting result may be derived if the intermolecular forces are assumed to be of a very short range near the beads $(i, j, \ldots)$. In that case the terms in the summation in the last term of (3.33) will differ from zero only if $\boldsymbol{r}_{a b} \simeq\left(\boldsymbol{r}_{i a}-\boldsymbol{r}_{a}\right)-\left(\boldsymbol{r}_{j b}-\boldsymbol{r}_{b}\right)$. Then, on writing $f_{a b}=\Sigma_{i j} f_{(i a)(j b)}$ it may be proved that

$$
\sum_{\substack{a, b \\ a, b \in p}} f_{a b} \boldsymbol{r}_{a b}=\sum \boldsymbol{f}_{i a}^{p a^{a}}\left(\boldsymbol{r}_{i a}-\boldsymbol{r}_{a}\right)
$$

in which

$$
f_{i a}^{p a^{\prime}}=\sum_{\substack{j b \\(b \neq a, b \in p)}} f_{(i a)(j b)} .
$$

From (3.33) and (3.35) we obtain

$$
T_{p}=-\frac{1}{V} \sum_{\substack{a \\ a \in p}} f_{a}^{\mathrm{B}} r_{a}+\frac{1}{V} \sum_{\substack{i, a \\ a \in p}} f_{i a}^{a^{\prime}}\left(\boldsymbol{r}_{i a}-\boldsymbol{r}_{a}\right)
$$

in which $f_{i a}^{a^{\prime}}=f_{i a}^{p^{\prime}}+f_{i a}^{p a^{\prime}}$ is the force acting on particle $i$ of molecule $a$ caused by interactions with particles in or outside the subset $p$ not belonging to the same molecule a. Averaging of the result (3.36) gives

$$
\boldsymbol{T}_{p}=\boldsymbol{T}^{\mathrm{B}}+n \sum_{i}\left\langle\boldsymbol{f}_{i}^{\prime}\left(\boldsymbol{r}_{i}-\boldsymbol{r}_{0}\right)\right\rangle
$$

This expression, in which $f_{i}^{\prime}$ is similar to $f_{i a}^{a^{\prime}}$, was first derived by Curtiss and Bird (1981) in their kinetic theory for polymer melts. 
This concludes our review of expressions for the macroscopic stress tensor in terms of microscopic variables in which we have focused the discussion mainly upon the consequences of the fundamental expression (3.7), derived from a virtual work principle. For other methods of derivation we refer especially to the textbook of Bird et al (1987b).

A very general and elegant expression for the reversible part of the stress tensor has been provided by Grmela $(1985,1986)$ on the basis of a bracket formulation of diffusion convection equations. This expression reads

$$
\boldsymbol{T}_{p}^{\mathrm{R}}=\frac{\delta A}{\delta \Phi} * \frac{\partial \dot{\Phi}^{-}}{\partial \boldsymbol{L}}
$$

Here $\Phi$ is a structural variable, like a tensor $\boldsymbol{S}$ or a configurational distribution function $\psi(\boldsymbol{q}, t) ; \dot{\Phi}$ is the material time derivative of $\Phi$ and $\dot{\Phi}^{-}$the part of $\dot{\Phi}$ that is odd in the sense that $\dot{\Phi}^{-}(\Phi, L)=-\dot{\Phi}^{-}(\Phi,-L)$ and $A=A\{\Phi\}$ is the free energy functional of the system. In general, the derivative $\delta A / \delta \Phi$ is a functional derivative and the multiplication $*$ an appropriate integration or contraction. In $\$ 7.2$ this expression will be discussed further for the case that $\Phi$ is a structure tensor.

\section{Bead-rod-spring models}

\subsection{Introduction}

The external forces on a polymer molecule in a dilute solution are the hydrodynamic forces due to the motion of the solvent. In bead-rod-spring models, the action of these forces is localised in a number of beads along the polymeric chain. The connectors between the beads may be rods or springs, in order to model stiff or elastic parts respectively of the chain. In general the chain may be branched and the connections between the rods may be constrained.

For a full analysis of general bead-rod-spring models we refer to chapter 16 of the textbook by Bird et al (1987b). In the next section we will illustrate some of the main features of these models by considering three special cases, the simplest case-the elastic dumbbell model - the bead-rod model as an example with a constraint and the Rouse-Zimm model in which a normal mode analysis is involved.

\subsection{Examples}

Since many features of general bead-rod-spring models are already present in the elastic dumbbell model, we start our discussion with a brief treatment of this model. In $\S 3.3$ we have already seen that the polymer contribution to the stress tensor may be represented in this case by the Kramers form (2.35). In order to derive a constitutive equation from this expression one needs information about the second moment $\langle\boldsymbol{q} q\rangle$ of the distribution function $\psi(\boldsymbol{q}, t)$. To this end we consider the evolution equation for $\psi$, which is based upon the continuity equation

$$
\frac{\partial \psi}{\partial t}=-\frac{\partial}{\partial \boldsymbol{q}} \cdot(\psi \dot{\boldsymbol{q}})
$$

and the equation of motion for the flux $\dot{q}$

$$
\dot{q}=L \cdot q-\frac{2 k T}{\zeta} \frac{\partial}{\partial q} \ln \psi-\frac{2 \kappa}{\zeta} q
$$


which follows from the equilibrium of forces (3.18) and the expressions (3.14), (3.16) and (3.17) for $f^{\mathrm{H}}, f^{\mathrm{B}}$ and $f^{1}$. Substitution of (4.2) in (4.1) gives an evolution equation of the Smoluchowski type (cf equation (3.1)). In this case, however, we do not need this equation. Instead, by multiplying (4.1) with the dyadic $q q$ and integration we obtain

$$
\frac{\mathrm{d}}{\mathrm{d} t}\langle\boldsymbol{q} \boldsymbol{q}\rangle=\langle\dot{\boldsymbol{q}} \boldsymbol{q}\rangle+\langle\boldsymbol{q} \dot{\boldsymbol{q}}\rangle
$$

and upon substitution of $\dot{\boldsymbol{q}}$ as given by (4.2)

$$
\frac{\delta}{\delta t}\langle q q\rangle=\frac{4 k T}{\zeta} 1-\frac{4 \kappa}{\zeta}\langle q q\rangle
$$

in which $\delta / \delta t$ denotes the upper convected derivative, defined in (A2.12). By eliminating $\langle q q\rangle$ from (3.20) and (4.4) we obtain a constitutive equation of the upper convected Maxwell type (cf equation (2.54))

$$
T_{p}+\lambda \stackrel{\nabla}{T}_{p}=2 n k T \lambda D
$$

with $\lambda=\xi / 4 \kappa$.

A derivation solely based upon a consideration of the second moment $\langle\boldsymbol{q q}\rangle$ is not possible if the spring-force modulus $\kappa$ is no longer a constant, but dependent upon $|\boldsymbol{q}|$. In that case, the substitution of (4.2) into (4.3) gives rise to a term containing the quantity $\langle\kappa q q\rangle$ which cannot be eliminated. A useful form of a nonlinear spring force law is the so-called FENE (finite extensible nonlinear elastic) expression

$$
f^{3}=\frac{\kappa q}{1-\left(q / q_{0}\right)} \quad q<q_{0} .
$$

An analysis and further references about the dumbbell model based upon this form may be found in the textbook of Bird et al (1987b).

Next we consider the bead-spring model, on which the classical theories of Rouse (1953) and Zimm (1956) on the viscoelastic behaviour of polymer solutions are based. (Our treatment closely follows the one of Lodge and Wu (1971).) In the bead-spring model a polymer chain is modelled by a set of $M$ beads at positions $\boldsymbol{r}_{1}, \boldsymbol{r}_{2}, \ldots \boldsymbol{r}_{M}$ connected by linear springs. The spring vectors will be denoted by $\tilde{r}_{1}, \tilde{r}_{2}, \ldots \tilde{r}_{M-1}$. The connection between these two sets of vectors is given by the equations

$$
G_{i j} \boldsymbol{r}_{j}=\tilde{\boldsymbol{r}}_{i}
$$

in which $G_{i j}=\delta_{i+1, j}-\delta_{i, j}$. We also define a centre $r_{0}$ by the equations

$$
H_{0 j} \boldsymbol{r}_{j}=\boldsymbol{r}_{0} \text {. }
$$

By requiring that $\Sigma_{j} H_{0 j}=1, r_{0}$ acquires the meaning of a weighted average of the bead positions. The matrix $H_{0 j}$ will be specified later on. The equations (4.7) and (4.8) will be abbreviated now as

$$
G r=\tilde{r} \quad H r=r_{0}
$$

and a similar matrix notation will be employed in the following treatment as long as possible.

The set of equations (4.9) may be inverted. So we also have the inverse transformation

$$
J \tilde{r}+K r_{0}=r
$$


The matrices $J$ and $K$, which are fully determined by $G$ and $H$ may be used now to define the forces $\tilde{f}$ and $f_{0}$ associated with any set of forces $\left(f_{1}, f_{2} \ldots f_{M}\right)=f$, acting upon the beads

$$
\tilde{f}=J^{\mathrm{T}} f \quad f_{0}=K^{\mathrm{T}} f
$$

By inversion of this set of equations, we also have

$$
f=G^{\mathrm{T}} \tilde{f}+H^{\mathrm{T}} f_{0} \text {. }
$$

Noting that for a virtual displacement $\delta r$ of the bead coordinates the virtual work becomes $\delta W=f \delta v=\delta r G^{\mathrm{T}} \tilde{f}+\delta r H^{\mathrm{T}} f_{0}=\tilde{f} \delta \tilde{r}+f_{0} \delta r_{0}$, we see that $\tilde{f}$ and $f_{0}$ may be considered as forces associated with the springs and the centre respectively.

Just as in the elastic dumbbell model where we needed an equation of motion for the flux $\dot{\boldsymbol{q}}$ in configuration space, we now need an expression for $\dot{\tilde{r}}$. This may be derived from the balance of forces

$$
f^{\mathrm{H}}+f=0 \text {. }
$$

Here, $f=f^{\mathrm{B}}+f^{1}$ with $f^{\mathrm{B}}$ the Brownian forces on the beads and $f^{1}$ the forces on the beads due to interactions (spring forces) with other beads. We now substitute $f^{\mathrm{H}}=$ $-\zeta(\dot{r}-v)$ in which $v$ represents the set of fluid velocities at the beads, obtaining

$$
-\zeta(\dot{r}-v)+f=0 \text {. }
$$

Now it becomes relevant to make a particular choice for the matrix $H$ (i.e. for the choice of the centre $r_{0}$ ). We take $H$ to be orthogonal to $G$ in the sense that

$$
G H^{\mathrm{T}}=0 \text {. }
$$

From the form of the $G$ matrix one readily shows that $H$ becomes

$$
H=\left[\frac{1}{M}, \frac{1}{M}, \frac{1}{M}, \ldots \frac{1}{M}\right] .
$$

So, in this case $r_{0}$ is the centre, in the usual sense. Later on we will see that in the case of hydrodynamic interaction between the beads this becomes different.

By multiplying (4.14) with $G$ and making use of (4.9), (4.12) and (4.13) we obtain

$$
\dot{\tilde{r}}=L \cdot \tilde{r}+\frac{A}{\zeta} \tilde{f}
$$

In this expression $A=G G^{\mathrm{T}}$ is the well known Rouse matrix

$$
A=\left(\begin{array}{rrrrr}
-2 & 1 & 0 & \ldots & 0 \\
-1 & 2 & -1 & \ldots & 0 \\
0 & 1 & 2 & -1 & 0 \\
0 & 0 & 0 & \ldots & 0 \\
0 & 0 & \ldots & -1 & 2
\end{array}\right)
$$

and the force $\tilde{f}=\tilde{f}^{\mathrm{B}}+\tilde{f}^{\mathrm{I}}$ where

$$
\tilde{f}^{\mathrm{B}}=-k T \frac{\partial}{\partial \tilde{r}} \ln \tilde{\psi}(\tilde{r}, t)
$$

and

$$
\tilde{f}^{\prime}=-\kappa \tilde{r}
$$


The equation (4.17) may be simplified by a transformation to normal coordinates. This is achieved by an orthogonal matrix $R: R R^{\mathrm{T}}=1$ which diagonalises $A: R A R^{\mathrm{T}}=\tilde{A}=$ $\operatorname{diag}\left(a_{1}, a_{2}, \ldots a_{M-1}\right)$ and transforms the coordinates and forces according to $\hat{r}=R \tilde{r}$, $\hat{f}=R \tilde{f}$. Then (4.17) becomes

$$
\dot{\hat{r}}_{i}=L \cdot \hat{r}_{i}+\frac{a_{i}}{\xi} \hat{f}_{i}
$$

(here we return again to the explicit notation).

The equation of continuity, transformed into normal coordinates, reads

$$
\frac{\partial \hat{\psi}}{\partial t}=-\frac{\partial}{\partial \hat{\boldsymbol{r}}_{i}} \cdot\left(\hat{\hat{\psi}} \dot{\hat{\hat{r}_{i}}}\right)
$$

Analogously to (4.4) one may derive from (4.21) and (4.22)

$$
\frac{\delta}{\delta t}\left\langle\hat{\boldsymbol{r}}_{i} \hat{\boldsymbol{r}}_{i}\right\rangle=\frac{2 a_{i} k T}{\zeta} 1-\frac{2 a_{i} k}{\zeta}\left\langle\hat{\boldsymbol{r}}_{i} \hat{\boldsymbol{r}}_{i}\right\rangle
$$

The stress tensor expression for the bead-spring model of the Kramers type (3.20) reads $T_{p}=\Sigma_{i} T_{i}$, with

$$
T_{i}=-n k T 1+n \kappa\left\langle\tilde{r}_{i} \tilde{r}_{i}\right\rangle
$$

This may readily be expressed in normal coordinates:

$$
T_{i}=-n k T 1+n \kappa\left\langle\hat{r}_{i} \hat{r}_{i}\right\rangle
$$

Now, from (4.23) and (4.25) we may eliminate the tensor $\left\langle\boldsymbol{r}_{i} \boldsymbol{r}_{i}\right\rangle$, obtaining the constitutive equation

$$
T_{i}+\lambda_{i} \stackrel{\nabla}{T}_{i}=2 n k T \lambda_{i} D
$$

with

$$
\lambda_{i}=\frac{\zeta}{2 a_{i} \kappa}
$$

So we see that the Rouse model leads to a set of upper-convected Maxwell equations with relaxation times, determined by the characteristic values $a_{i}$ at the matrix $A$.

It is evident that for nonlinear (branched, star-shaped, etc) model structures the matrix $G$ and so the matrix $A$ will be different while most of the analysis outlined above will remain more or less the same. In that case the set of characteristic values $\left\{a_{i}\right\}$ and so the relaxation spectra will be different. Other modifications are the introduction of different friction coefficients for different beads and/or different spring constants for different springs. This may be included in the present formalism by the introduction of matrices $G_{i j}$ and/or $\kappa_{i j}$ instead of the scalar coefficients $G$ and/or $\kappa$ used above and some minor modifications in the analysis. Also in that case the main effect will be a change of the relaxation spectrum.

One modification that will be discussed now in more detail is the inclusion of hydrodynamic interaction. This, in fact, is the improvement to the Rouse theory, given by Zimm (1956). The point is that in the expression for the hydrodynamic forces $f_{i}^{\mathrm{H}}$, used so far, we took $v_{i}=v_{0}+L \cdot\left(r_{i}-r_{0}\right)$ as the velocity of the solvent at the beads, in applying Stokes' law. In fact, the velocities $v_{i}$ at the beads are also determined by the 
disturbance of the flow field due to the other beads. If this disturbance is included, the hydrodynamic forces become

$$
f_{i}^{\mathrm{H}}=-G\left(\dot{\boldsymbol{r}}_{i}-\boldsymbol{v}_{i}+\sum_{j} \boldsymbol{\Omega}_{i j} f_{j}\right)
$$

in which $\boldsymbol{\Omega}_{i j}$ is the Oseen tensor, determining the disturbance of the velocity field at $\boldsymbol{r}_{i}$ due to the force $f_{j}$ acting at $\boldsymbol{r}_{j}$. Usually a pre-averaging is used, in which $\boldsymbol{\Omega}_{i j}$ is replaced by its average $\left\langle\boldsymbol{\Omega}_{i j}\right\rangle_{0}$ with respect to the (Gaussian) equilibrium distribution $\psi_{0}\left(\boldsymbol{r}_{1}, \boldsymbol{r}_{2}, \ldots \boldsymbol{r}_{M}, t\right)$. Analogous to (4.14) we now obtain from the equilibrium of forces

$$
-G(\dot{r}-v)+Z f=0 \text {. }
$$

Here we have used again the abbreviated notation, in which $Z$ denotes the matrix

$$
\boldsymbol{Z}_{i j}=\delta_{i j}+\left(1-\delta_{i j}\right) \xi\left\langle\boldsymbol{\Omega}_{i j}\right\rangle_{0} .
$$

Explicit expressions of $\boldsymbol{\Omega}_{i j},\left\langle\boldsymbol{\Omega}_{i j}\right\rangle_{0}$ and $\boldsymbol{Z}_{i j}$ can be found in $\S 15.4$ of Bird et al (1987b). Following the previous analysis of the Rouse model we introduce a matrix $G$ and spring vectors $\tilde{r}$ as before, but instead of $H$ a different matrix $H^{\prime}$ (to be determined later on). Introducing in (4.29) an expression for $f$ analogous to (4.12) and multiplication by $G$ gives

$$
-\xi(\dot{\tilde{r}}-L \cdot \tilde{r})+G Z H^{\prime \top} f_{0}^{\prime}+G Z G^{\mathrm{T}} \tilde{f}=0 .
$$

We now use the freedom in the choice of $H^{\prime}$ by requiring

$$
G Z H^{\prime T}=0 \text {. }
$$

By this equation $H^{\prime}$ is uniquely defined. The associated centre $r_{0}^{\prime}=H^{\prime} r$ is called the centre of resistance of the molecule.

From (4.31) and (4.32) we obtain

$$
\tilde{r}=L \cdot \tilde{r}+\frac{A^{\prime}}{\xi} \tilde{f}
$$

with $A^{\prime}=G Z G^{\mathrm{T}}$, a modified Rouse matrix. This equation is completely similar to (4.17) and so is the subsequent analysis. The Zimm theory, therefore, yields the same form (4.26) of constitutive equation as the Rouse theory. Only the relaxation spectrum will be different, because of the difference between the matrices $A$ and $A^{\prime}$.

Experimental evidence shows that the inclusion of hydrodynamic interaction indeed gives a significant improvement of the predictions of the theory as compared with experimental data (especially on linear viscoelastic response) of dilute polymer solutions. Strangely enough, the Rouse model-although intended for dilute systems only-often gives better predictions for more concentrated systems. This may be caused by the shielding of hydrodynamic interaction.

We now continue our overview of bead-rod-spring models by considering the effect of constraints. In general, the (holonomic) constraints may be represented by a set of equations

$$
C_{p}\left(\boldsymbol{r}_{1} \ldots \boldsymbol{r}_{M}\right)=0 \quad p=(1,2, \ldots 3 M-f)
$$

where $f$ is the number of degrees of freedom of the system.

Constraints can be handled by two methods (see also Doi and Edwards (1986, §3.8)). 
(i) The method, adopted by Kirkwood $(1949,1967)$ in which the motion of the model structure is described by a set of independent generalised coordinates $Q_{i}$ $(i=1,2, \ldots f)$. This method has also been employed in the general treatment of arbitrary bead-rod-chain systems in chapter 16 of the textbook by Bird et al (1987b).

(ii) A method, used by Fixman $(1974,1975)$ in which the motion of the model structure is still described by the set of bead vectors $\left\{\boldsymbol{r}_{i}\right\}$ in the full $3 \mathrm{M}$-dimensional configuration space but constrained by the equations (4.34). In the equation of motion constraining forces then appear which may be evaluated by the method of Lagrangian multipliers.

One may also apply a method based upon projection operators like the operator $\boldsymbol{P}$ associated with the rigidity constraint in the rigid dumbbell model discussed in $\S 3.2$. This method will be illustrated now. Referring to the literature cited above for a full discussion of constrained bead-rod-spring systems we will restrict the discussion here to the rigid dumbbell model. For this model the force $f^{1}$ becomes a constraining force in the direction of the unit vector $e=q /|\boldsymbol{q}|$. So if we multiply the balance of forces analogous to (3.18) with the projection operator $\boldsymbol{P}$ defined in (3.22) the term $\boldsymbol{P} \cdot \boldsymbol{f}^{\mathrm{I}}$ will vanish and analogously to (4.2) we obtain

$$
\boldsymbol{P} \cdot \dot{q}=\boldsymbol{P} \cdot\left(\boldsymbol{L} \cdot \boldsymbol{q}-\frac{2 k T}{\zeta} \frac{\partial}{\partial \boldsymbol{q}} \ln \psi\right)
$$

or, since $\boldsymbol{P} \cdot \dot{\boldsymbol{q}}=\dot{\boldsymbol{q}}=q \dot{\boldsymbol{e}}$, and $q \boldsymbol{P} \cdot \partial / \partial \boldsymbol{q}=\partial / \partial \boldsymbol{e}$ the rotation gradient operator, also

$$
\dot{e}=(1-e e) \cdot L \cdot e-\frac{2 k T}{\zeta q^{2}} \frac{\partial}{\partial e} \ln \psi
$$

This equation determines the rotational flux, which has to be substituted into the rotational continuity equation

$$
\frac{\partial \psi}{\partial t}=-\frac{\partial}{\partial \boldsymbol{e}}(\psi \dot{\boldsymbol{e}})
$$

to give an evolution (Smoluchowski) equation for the distribution function $\psi$. With a solution of this equation, which in most cases has to be determined numerically, the stress tensor may be calculated by performing the averages in the expression (3.25). In this case it is not possible to derive an explicit constitutive equation by eliminating the moments $\langle e e\rangle$ and $\langle e e e\rangle$ since no evolution equations exist from which together with the stress tensor expression both moments can be eliminated simultaneously.

\section{Transient-network models}

\subsection{Introduction}

Many theories about the rheological behaviour of melts and concentrated solutions are based upon the transient-network concept, originally developed by Green and Tobolsky (1947), Lodge (1956) and Yamamoto (1956). For a review of these theories we refer to the paper by Lodge et al (1982) and to chapter 20 of the book by Bird et al (1987b). In the present paper we will not enter into the details of any particular model, but merely discuss some general aspects of this type of model. 
The transient-network model is proposed to describe a concentrated polymer solution or melt. In such a system the molecules will have many interactions with each other. A number of these will have the character of an entanglement and behave like a temporary bond. An idealised picture of a system like this is a network, consisting of Gaussian segments which are created and annihilated at certain rates. The polymer contribution to the stress tensor of this system is given by (see also equation (3.30))

$$
T_{p}=n \kappa\langle q q\rangle
$$

in which $n$ is the number density of segments, $q$ the segment vector and the angular brackets denote an average with respect to the (one)-segment distribution function $\psi(q, t)$. The further specification of any transient-network model requires the specification of the kinetics of creation and destruction of segments, of the evolution of the distribution function and some other properties of the transient network. These will be discussed in the next section.

\subsection{The Lodge model}

In this section we will give an outline of the common structure of most transient network theories for polymeric liquids. Although our treatment will be somewhat different and slightly more general, it is essentially an outline of the theory of Lodge (1956).

We start with the expression (5.1) of the stress tensor. In a more explicit notation this equation reads

$$
T_{p}=n \kappa \int \psi(q, t) q q \mathrm{~d}^{3} q .
$$

In order to develop this expression further, more information is needed about the evolution of the distribution function $\psi$. In the transient-network model this evolution is determined by creation and loss processes and by the affine convection of segments. This may be summarised in mathematical form as follows

$$
\psi(\boldsymbol{q}, t)=\int_{-\infty}^{t} \int \mu\left(t, t^{\prime}\right) \delta\left(\boldsymbol{q}-\overline{\boldsymbol{q}}\left(\boldsymbol{q}^{\prime}, t^{\prime}, t\right)\right) \hat{\psi}\left(\boldsymbol{q}^{\prime}\right) \mathrm{d}^{3} \boldsymbol{q}^{\prime} \mathrm{d} t^{\prime} .
$$

In this expression $\mu\left(t, t^{\prime}\right) \mathrm{d} t^{\prime}$ is the probability per segment, present at time $t$, of being created at time $t^{\prime}, \hat{\psi}\left(\boldsymbol{q}^{\prime}\right) \mathrm{d}^{3} \boldsymbol{q}$ the probability per segment created at $t^{\prime}$ of being created with its segment vector in an element $d^{3} q$ around $q^{\prime}$ and

$$
\bar{q}\left(q^{\prime}, t^{\prime}, t\right)=F_{t}^{-1}\left(t^{\prime}\right) \cdot q^{\prime}
$$

expressing the assumption of the affine motion of the segments.

In the theory of Lodge the creation distribution $\hat{\psi}\left(\boldsymbol{q}^{\prime}\right)$ is taken to be equal to the Gaussian equilibrium distribution, so we have

$$
\left\langle q^{\prime} q^{\prime}\right\rangle^{\prime}=\frac{k T}{\kappa} 1
$$

in which \langle\rangle$^{\prime}$ denotes an average with respect to $\hat{\psi}\left(\boldsymbol{q}^{\prime}\right)$.

We now substitute the expression (5.3) for $\psi$ in (5.2). By performing the integrations with respect to $q$ and $q^{\prime}$, on using (5.4) and (5.5) we obtain

$$
T_{p}=n k T \int_{-\infty}^{t} \mu\left(t, t^{\prime}\right) C_{r}\left(t^{\prime}\right)^{-1} \mathrm{~d} t^{\prime}
$$


This integral expression already shows some features of the transient-network model. The $C_{t}^{-1}$ tensor appears as a result of the assumption (5.4) of affine motion. In order to get more specific results we have to look closer at the kinetics of creation and loss of segments. First we define a function

$$
\tilde{n}\left(t, t^{\prime}\right)=n(t) \mu\left(t, t^{\prime}\right)
$$

being the number of segments created per unit time at $t^{\prime}$ and still existing at time $t$. Since $\int_{-\infty}^{t} \mu\left(t, t^{\prime}\right) \mathrm{d} t^{\prime}=1$, as can be seen by integrating (5.8) with respect to $q$ it follows that

$$
\int_{-\infty}^{t} \tilde{n}\left(t, t^{\prime}\right)=n(t)
$$

Furthermore we define the creation rate

$$
g\left(t^{\prime}\right)=\frac{1}{n_{0}} \tilde{n}\left(t^{\prime}, t^{\prime}\right)
$$

in which $n_{0}$ is the segment density at equilibrium, and an annihilation rate $h(t)$ by

$$
\frac{\partial}{\partial t} \tilde{n}\left(t, t^{\prime}\right)=-h(t) \tilde{n}\left(t, t^{\prime}\right)
$$

By differentiating the expression (5.8) with respect to $t$ (at constant $t^{\prime}$ ) and making use of $(5.7),(5.9)$ and $(5.10)$ we obtain a rate equation for the total number of segments

$$
\frac{\mathrm{d} n}{\mathrm{~d} t}=g n_{0}-h n
$$

or, expressed by the ratio $x=n / n_{0}$,

$$
\frac{\mathrm{d} x}{\mathrm{~d} t}=g-h x .
$$

From (5.9) and (5.10) a formal solution for $\tilde{n}$ may be derived. If this solution is substituted in (5.6) one obtains the constitutive equation

$$
\boldsymbol{T}_{p}=G_{0} \int_{-\infty}^{t} g\left(t^{\prime}\right) \exp \left(-\int_{t^{\prime}}^{t} h\left(t^{\prime \prime}\right) \mathrm{d} t^{\prime \prime}\right) C_{t}^{-1}\left(t^{\prime}\right) \mathrm{d} t
$$

in which $G_{0}=n_{0} k T$.

In order to obtain more explicit constitutive equations the creation and loss functions $g$ and $h$ have to be specified. In general these quantities may depend upon macroscopic flow variables, like the invariants of the rate of strain tensor, or of strain tensors like $C_{t}^{-1}\left(t^{\prime}\right)$. Sometimes a stress dependence is assumed. In that case the expressions (5.6) and (5.13) become integral equations instead of explicit expressions for $T_{E}$.

In the simplest case (the one corresponding to the original formulation by Lodge) one takes $g$ and $h$ to be constant. Then (5.11) implies that $g=h, n=n_{0}$ and the expression (5.13) reduces to

$$
T_{p}=G_{0} \int_{-\infty}^{t} \frac{1}{\lambda} \exp \left(-\frac{\left(t-t^{\prime}\right)}{\lambda}\right) C_{t}^{-1}\left(t^{\prime}\right) \mathrm{d} t^{\prime}
$$

with $\lambda=g^{-1}=h^{-1}$. 
We now return again to the fundamental expression (5.3) for the evolution of $\psi$. On differentiating this expression to $t$ and writing the time derivative of the delta function as

$$
\frac{\partial}{\partial t} \delta(\boldsymbol{q}-\overline{\boldsymbol{q}})=\frac{\partial \delta(\boldsymbol{q}-\overline{\boldsymbol{q}})}{\partial \overline{\boldsymbol{q}}} \cdot \frac{\partial \overline{\boldsymbol{q}}}{\partial t}=-\frac{\partial}{\partial \boldsymbol{q}} \cdot\{\delta(\boldsymbol{q}-\overline{\boldsymbol{q}}) \dot{\boldsymbol{q}}\}
$$

the following differential equation for $\psi$ is obtained

$$
\frac{\partial \psi}{\partial t}=-\frac{\partial}{\partial \boldsymbol{q}} \cdot(\psi \dot{\boldsymbol{q}})+\frac{g}{x}(\hat{\psi}-\psi) .
$$

Here we used the fact that $\mu(t, t)=g / x$ and the relation $\partial \mu\left(t, t^{\prime}\right) / \partial t=-(g / x) \mu\left(t, t^{\prime}\right)$ which can be proved by differentiation of the normalisation condition $\int_{-\infty}^{t} \mu\left(t, t^{\prime}\right) \mathrm{d} t=1$. From (5.16) one may readily obtain a differential equation for the total segment distribution $\Psi(q, t)=n \psi(q, t)$. This equation becomes

$$
\frac{\partial \Psi}{\partial t}=-\frac{\partial}{\partial \boldsymbol{q}} \cdot(\Psi \dot{\boldsymbol{q}})+g \hat{\Psi}_{0}-h \Psi
$$

with $\hat{\Psi}_{0}=n_{0} \hat{\psi}$.

This equation is usually proposed directly in transient-network models since it has an obvious heuristic interpretation: The rate of change of the total distribution function $\Psi$ of all segments changes as a result of convection (the first term in the right-hand side of (5.17)), creation (the second term) and loss (the last term). Creation of segments takes place in the equilibrium distribution $\hat{\Psi}_{0}=n_{0} \hat{\psi}$ and the loss of segments is proportional to the actual distribution $\Psi$.

A constitutive equation may readily be obtained from (5.17) by multiplication with $q q$ and integration. On using again the stress-tensor expression (5.1), the assumption of affine motion (5.4) which in differential form becomes

$$
\dot{q}=\boldsymbol{L} \cdot \boldsymbol{q}
$$

and (5.5) for the average with respect to $\hat{\psi}$ one obtains an equation of the upperconventional Maxwell type (cf $\$ 2.4$ )

$$
T_{p}+\lambda \stackrel{\nabla}{T}_{p}=G 1
$$

(with $\lambda=h^{-1}$ and $G=\lambda g G_{0}$ ).

This equation may be shown to be equivalent to the integral forms (5.6) and (5.13). Since we assume incompressibility, an arbitrary isotropic stress may be added to $T_{p}$ (see $\S 2.2$ ). If, for this purpose, the stress $-n k T 1$ is used we obtain from (5.19) the equivalent form

$$
T_{p}+\lambda \stackrel{\nabla}{T}_{p}=2 \eta D
$$

(with $\lambda=h^{-1}$ and $\eta=\kappa G_{0} \lambda=n k T \lambda$ ) which is similar to the constitutive equation (4.5) of the dumbbell model.

This concludes our general outline of the Lodge model. In the next section we will discuss some special cases and generalisations of this model.

\subsection{Generalisations}

The transient-network model offers attractive possibilities for a semi-empirical modelling of the rheological behaviour of polymeric liquids. By making specific choices 
for the kinetic functions $g$ and $h$ and introducing additional modifications (to be discussed below) various models may be generated. We now will discuss some examples of such modifications.

First we note the possibility of introducing a relaxation spectrum $\left\{\lambda_{i}, G_{i}\right\}$ instead of one relaxation strength $G_{0}$ as has been used so far. This is usually done by considering a number of different kinds of segments, identified by a number $i$, called the complexity. Then we have

$$
T_{p}=\sum T_{i}
$$

with $T_{i}$, given by a constitutive equation similar to (5.20),

$$
\boldsymbol{T}_{i}+\lambda_{i} \stackrel{\nabla}{\boldsymbol{T}}_{i}=2 \lambda_{i} G_{i} \boldsymbol{D}
$$

with $G_{i}=n_{i} k T, \lambda_{i}=h_{i}^{-1}$ and $\mathrm{d} n_{i} / \mathrm{d} t=g_{i}-h_{i} n_{i}$.

The relaxation spectrum is often left unspecified; in some cases (Meister 1971, Carreau 1972, De Kee and Carreau 1979) one uses the Rouse spectrum

$$
\lambda_{i}=\frac{2^{\alpha} \lambda_{i}}{(i+1)^{\alpha}} ; G_{i}=G \quad(i=1,2,3, \ldots)
$$

determined by three parameters: $\alpha_{i}, \lambda_{i}$ and $G$ or some special form of the same type (Bird and Carreau 1968). Contrary to the Rouse model (see $\$ 4.2$ ) where the relaxation spectrum was derived, expressions like (5.23) must be considered as purely empirical, since the transient-network model offers no possibility of deriving a relaxation spectrum.

A second generalisation of the model, described in the preceding section, is the introduction of non-affine convection of the segments. In that case the function $\bar{q}\left(\boldsymbol{q}^{\prime}, t^{\prime}, t\right)$ in (5.3) is not given by (5.4) but by some other convection law. A well known type of non-affine convection, proposed by Phan-Thien and Tanner (1977), is of the type

$$
\overline{\boldsymbol{q}}\left(q^{\prime}, t^{\prime}, t\right)=\tilde{\boldsymbol{F}}_{t}^{-1}\left(t^{\prime}\right) \cdot \boldsymbol{q}^{\prime}
$$

In this expression $\tilde{F}_{t}\left(t^{\prime}\right)$ is an effective deformation gradient tensor, different from the tensor $\boldsymbol{F}_{t}\left(\boldsymbol{t}^{\prime}\right)$. Corresponding to $(5.18)$ we have

$$
\dot{q}=\tilde{L} \cdot q
$$

in which

$$
\tilde{\boldsymbol{L}}=\left.\frac{\partial}{\partial t^{\prime}} \tilde{F}_{t}\left(t^{\prime}\right)\right|_{t^{\prime}=t}
$$

is an effective velocity gradient tensor. In the Phan-Thien-Tanner model one assumes

$$
\tilde{L}=\boldsymbol{L}-\boldsymbol{\xi} \boldsymbol{D} \quad(0 \leqslant \zeta<1) .
$$

A similar effective velocity gradient (see also Appendix 2) was introduced earlier in a modified dumbbell model by Gordon and Schowalter (1972); it was used also by Johnson and Segalman $(1977,1981)$ in a phenomenological model.

It is not difficult to incorporate this type of non-affine convection in the transientnetwork theory. The only changes are that the Finger tensor $C_{t}^{-1}\left(t^{\prime}\right)$ in $(5.6)$ and $(5.13)$ becomes $\tilde{C}_{t}^{-1}\left(t^{\prime}\right)$, based upon $\tilde{F}_{t}\left(t^{\prime}\right)$, defined in (5.24) and that everywhere in the analyses the velocity gradient $\boldsymbol{L}$ should be replaced by $\tilde{\boldsymbol{L}}$ ( $\boldsymbol{D}$ by $\tilde{\boldsymbol{D}}$, etc). The convected derivative in (5.19) then becomes the Gordon and Schowalter derivative $\widetilde{T}$, defined by (A2.16). The resulting constitutive equation is of the form (2.58) with $\sigma=1$. 
Non-affine convections of type different from (5.25) are far more difficult to handle in transient-network theory. Examples are (see Larson 1983) non-extending non-affine convection

$$
\dot{q}=L \cdot q-(e \cdot D \cdot e) q
$$

which is also relevant to reptation models $(\S 6)$, and partially extending non-affine convection

$$
\dot{q}=L \cdot q-\xi(e \cdot D \cdot e) q
$$

used in a theory of polymer melts by Larson (1984) and in a transient network theory of concentrated dispersions by Kamphuis et al (1984), and the quasi-linear non-affine convection

$$
\dot{q}=q \cdot Z: L
$$

(in which $\boldsymbol{Z}$ is a fourth-order tensor), discussed by Larson $(1983,1985)$.

The assumptions about the kinetics of transient-network models are contained in the expressions of the coefficients $g$ and $h$ in (5.13). We have already discussed the simplest case (Lodge 1956) in which $g$ and $h$ are constant. This case is attractive for its simplicity but not very realistic in its predictions of rheological behaviour.

A first type of modification of the kinetics of the Lodge model are models with rate of strain dependent functions $g$ and $h$. The objectivity and symmetry requirements of simple fluid theory require these functions to be isotropic. Usually one assumes dependence upon the second invariant of the rate of strain tensor

$$
g=g\left(I I_{D}\right) \quad h=\left(I I_{D}\right) .
$$

Theories of this type have been proposed by Bogue (1966), Bird and Carreau (1968), Meister (1971) and Carreau (1972). As an example we give the kinetic functions of a model by Meister (1971)

$$
g_{i}=\frac{1}{\lambda_{i}} \quad h_{i}=\frac{1}{\lambda_{i}}\left(1+c \lambda_{i} \sqrt{I I_{D}}\right)
$$

in which $\lambda_{i}$ is the Rouse spectrum (5.23). The kinetic coefficients may also depend upon the macroscopic stress. Usually the excess stress $T^{\prime}=T_{E}-T_{0}$ with respect to the equilibrium value $T_{0}$ is used for this purpose and a dependence of the first invariant $I_{T^{\prime}}=\operatorname{tr} T^{\prime}$ is assumed:

$$
g=g\left(\operatorname{tr} T^{\prime}\right) \quad h=h\left(\operatorname{tr} T^{\prime}\right)
$$

Examples of models with this type of kinetics are the models of Kaye (1966) and of Phan-Thien and Tanner (1977). In the latter model, the kinetic functions are given by

$$
g_{i}=h_{i}=\frac{1}{\lambda_{i}}\left(1+\varepsilon \frac{\operatorname{tr} T_{i}^{\prime}}{g_{0 i}}\right)
$$

in which $\varepsilon$ is a parameter.

In some models the creation and annihilation functions are assumed to depend upon both the stresses $T_{i}^{\prime}$ and on the segment densities $n_{i}$, or equivalently the concentration ratios $x_{i}=n_{i} / n_{i 0}$, so

$$
g_{i}=g_{i}\left(x_{i}, T_{i}^{\prime}\right) \quad h_{i}=h_{i}\left(x_{i}, T_{i}^{\prime}\right)
$$


Examples of this type are the model of Acierno et al (1976) often called the 'Marrucci model' and the model of Soong and Shen (1979). Both models were originally not formulated as transient-network models, but it has been proved (Jongschaap 1981, Doeksen et al 1985) that such a formulation is still possible. The kinetic functions of the Marrucci model are

$$
\begin{aligned}
& g_{i}=\frac{1}{\lambda_{i 0} x_{i}^{0,4}} \\
& h_{i}=\frac{1}{\lambda_{i 0} x_{i}^{1,4}}\left[a\left(\frac{\operatorname{tr} T_{i}}{2 G_{0 i} x_{i}}\right)^{1 / 2}-\frac{1}{x_{i}}+2\right]
\end{aligned}
$$

in which $a$ is a parameter.

Instead of assuming a dependence upon macroscopic flow properties, like $D, T$ and $x$, it seems more natural to assume a dependence of the kinetic functions upon the segment vector $q$ :

$$
g=g(q) \quad h=h(q) .
$$

This assumption was made already in the theory of Yamamoto (1956) which was developed at the same time as the Lodge (1956) model. For detailed considerations about the molecular foundations of the assumptions in this theory we refer to the papers of Wiegel (1969) and Wiegel and de Bats (1969).

Although the results of the theory of Yamamoto are promising, at least for small shear rates, this type of modelling has not become very popular in transient-network theory. The main difficulty is that these models cannot be solved analytically and that no constitutive equation can be derived. Recently, however, a numerical approach has been presented (Petruccione and Biller 1988) which allows the solution of transientnetwork models of the Yamamoto type with little computational effort. Although some of their results are promising their conclusion is that the introduction of configurationaldependent creation and loss rates does not seem to represent a real improvement in fitting experimental data with transient-network models. They suggest that probably an extended approach to network theories is needed, which takes into account the cooperative dynamics of the creation and loss of segments.

All modifications and generalisations of the Lodge model introduced so far may be formulated in the integral form (5.13) as well as in the differential form (5.19) of the model (although as already mentioned the integral formulation of models with stress-dependent kinetic functions is not very useful, since in that case the constitutive equation becomes an integral equation).

An exception is a modification, first introduced by Wagner. This modification which can only be applied to the integral form consists of the introduction of a factor $\mathscr{H}\left(t, t^{\prime}\right)$, the so-called damping function in the integrand. In that case (5.13) becomes

$$
T_{p}=G_{0} \int_{-\infty}^{t} g\left(t^{\prime}\right) \mathscr{H}\left(t, t^{\prime}\right) \exp \left(-\int_{t^{\prime}}^{t} h\left(t^{\prime \prime}\right) \mathrm{d} t^{\prime \prime}\right) C_{t}^{-1}\left(t^{\prime}\right) \mathrm{d} t^{\prime} .
$$

In the theory of Wagner the damping function is assumed to depend upon the first principal invariants of the Finger tensor, $I=\operatorname{tr} C_{t}^{-1}\left(t^{\prime}\right)$ and $I I=\operatorname{tr} C_{t}\left(t^{\prime}\right)$, so

$$
\mathscr{H}=\mathscr{H}(I, I I) \text {. }
$$

In the case that the kinetic functions $g$ and $h$ are constant (and also in some other cases) the constitutive equation (5.38) then becomes of the $\mathrm{K}-\mathrm{BKZ}$ type $((2.62)$ and 
(2.63)). An example of a damping function is the one proposed by Wagner (1976)

$$
\mathscr{H}\left(t, t^{\prime}\right)=\exp [-(n \cdot \delta) \sqrt{I I-3}-\delta \sqrt{I-3}]
$$

in which $n$ and $\delta$ are dimensionless parameters. In order to improve the model, especially with respect to the description of recovery following start-up of steady elongational flow, Wagner and Stephenson (1979) modified their theory by replacing the damping function $\mathscr{H}$ by a functional. For further details we refer to their paper.

\section{Reptation models}

\subsection{Introduction}

Although the transient-network concept, discussed in $\$ 5$, offers attractive facilities for the description of the rheological properties of concentrated polymer solutions and melts, this type of modelling is not entirely satisfactory. One of the reasons is that the transient-network model is not really a molecular model. Therefore instead of being expressed in molecular quantities like molecular mass, chain branching, etc its results are merely expressed in unspecified model parameters.

A class of models which are closer to the molecular reality are the so-called reptation models. These models are based upon the concept of reptation, originally proposed by de Gennes (1971) and used in a rheological model by Doi and Edwards (1978, 1979 ) and by Curtiss and Bird (1981) in a different way. As already stated in $\$ 3.1$, reptation models are mean field theories, in which the environment of a molecule is modelled in some way in order to represent the topological constraints due to the neighbouring molecules and causing a reptating motion of the central molecule.

In $\S \S 6.2$ and 6.3 we will discuss the two main approaches in this field. First in $\S 6.2$ the tube model, used by Doi and Edwards $(1978,1979)$ and in $\S 6.3$ the Curtiss-Bird theory (1981).

\subsection{Tube models}

The tube model in polymer rheology was first introduced by de Gennes (1971) who discussed the Brownian motion of an unattached polymer chain through a fixed network. He noted that the motion of the chain was always as if it were confined in a tube and that as a result of this motion the tube itself changes with time. Doi and Edwards $(1978,1979)$ used this concept in a model for the rheological behaviour of concentrated polymer solutions and melts.

In their model the tube consists of $N$ segments, each of a length $a$ and a total length $L=N a$. The size of the diameter of the tube is also of order $a$ and the molecule is treated as a Gaussian chain, confined in the tube. The average contour of the molecular chain coincides with the centre line of the tube and is called the "primitive chain'. Due to the thermal motion of the molecule, the primitive chain will perform a diffusive motion in the tube along its own contour (reptation). Only at the ends of the tube may the chain (we will often use the word chain instead of 'primitive chain') choose arbitrary new directions, creating new randomly oriented tube elements. At the opposite end of the tube, at the same time, tube elements wil disappear since the chain is moving out. 
The motion of the primitive chain in the tube is a diffusion process, governed by a diffusion equation. The probability densities by which the process of creation and loss of tube elements is described are determined by similar diffusion equations. In particular the probability density $\Phi\left(t-t^{\prime}, s\right)$ of a tube element present at time $t^{\prime}$ and still present at time $t$ at a curvilinear position $s$ along the primitive chain is determined by the diffusion equation

$$
\frac{\partial \Phi}{\partial t}=D \frac{\partial^{2} \Phi}{\partial s^{2}}
$$

under the boundary conditions $\Phi\left(t-t^{\prime}, 0\right)=\Phi\left(t-t^{\prime}, L\right)=0$ and $\Phi(0, s)=1$. The diffusion constant $D$ is given by the Einstein relation

$$
D=\frac{k T}{L \zeta}
$$

in which $\zeta$ is a friction coefficient per unit length of the primitive chain. We also need the function $\chi\left(t-t^{\prime}, s\right)=(\partial / \partial t) \Phi\left(t-t^{\prime}, s\right)$, which may be considered as the probability density for a tube element, created at time $t^{\prime}$ to survive and to be at a position $s$ along the primitive chain at time $t$. This function is given by the following expression

$$
\chi\left(t-t^{\prime}, s\right)=\sum_{p \text { (odd) }} \frac{4 p}{\pi \tau_{\mathrm{d}}} \sin \frac{p n s}{L} \exp \left(-\frac{\left(t-t^{\prime}\right) p^{2}}{\tau_{\mathrm{d}}}\right)
$$

where

$$
\tau_{\mathrm{d}}=\frac{L^{2}}{\pi^{2} D}
$$

is a time constant, the so called disengagement time.

Besides the diffusive motion, causing the creation and loss of tube elements, there is also a convective motion. It is assumed that the tube segments move affinely with the macroscopic flow. In particular, a unit vector $e$ along a tube element will obey the convection law

$$
e=\bar{e}\left(e^{\prime}, t^{\prime}, t\right)=\frac{F_{t^{\prime}}(t) \cdot e^{\prime}}{\left|F_{t^{\prime}}(t) \cdot e^{\prime}\right|}
$$

which implies for its rate of change

$$
\dot{e}=L \cdot e-D: e e \text {. }
$$

Analogous to equation (5.3) of the transient-network model, a similar equation holds for the probability density $\psi(e, t, s)$ of a tube element for having an orientation $e$ at time $t$ at a position $s$ along the chain. This equation becomes

$$
\psi(e, t, s)=\int_{-\infty}^{t} \int \chi\left(t-t^{\prime}, s\right) \delta\left(e-\bar{e}\left(e^{\prime}, t^{\prime}, t\right)\right) \hat{\psi}\left(e^{\prime}\right) \mathrm{d}^{2} e^{\prime} \mathrm{d} t^{\prime}
$$

in which the function $\bar{e}$ is given by (6.5) and the creation distribution function $\hat{\psi}=1 / 4 \pi$ corresponds with the assumption of random orientations of creation of new tube elements. Similar to (5.16) it may be shown that $\psi$ obeys the evolution equation

$$
\frac{\partial \psi}{\partial t}=-\frac{\partial}{\partial \boldsymbol{e}} \cdot(\psi \dot{e})+D \frac{\partial^{2} \psi}{\partial s^{2}}
$$

in which $\dot{e}$ is given by (6.6). 
The stress tensor is calculated from an equation of the type (3.11) in which the average with respect to the whole chain is changed into $N$ times an average with respect to the one-segment distribution function $\psi(e, s, t)$, so

$$
T_{p}=n N \int_{0}\langle\bar{\sigma} e e\rangle \mathrm{d} s .
$$

In this expression $\bar{\sigma}=\sigma / N$ is the contribution of one segment to the stress $\sigma$ in the chain. The approximation used in (6.9) is essentially the so-called independent alignment approximation introduced by Doi and Edwards, which states that the convective motion of segments of a primitive chain segment is given by (6.5), irrespective of its motion along the tube. This enables the simple analysis based upon a one-segment distribution given below.

The stress $\sigma$ in the chain is calculated by Doi and Edwards from the force boundary conditions at the end of the tube, which are necessary in order to keep the monomer density per unit length of a Gaussian chain at its equilibrium value. The result is $\sigma=3 k T / a$. (This is also the entropic force, $f=\left(3 k T / M b^{2}\right) a$, needed to keep the end points of a segment consisting of $M$ monomer units of length $b$ at a distance $a=\sqrt{M b^{2}}$.) The contribution per segment $\bar{\sigma}=\sigma / N$ to the equilibrium stress also becomes

$$
\bar{\sigma}=\frac{3 k T}{L} \text {. }
$$

A constitutive equation may be obtained now by substituting (6.10) in (6.9) and performing the averaging with respect to $\psi(e, t, s)$, given by $(6.7)$. In this way we obtain

$$
T_{p}=3 n N k T \int_{-\infty}^{t} \mu\left(t-t^{\prime}\right)\langle\bar{e} \bar{e}\rangle^{\prime} \mathrm{d} t^{\prime}
$$

in which

$$
\mu\left(t-t^{\prime}\right)=\frac{1}{L} \int_{0}^{L} \chi\left(t-t^{\prime}, s\right) \mathrm{d} s=\sum_{p(\text { odd })} \frac{8}{\pi^{2} \tau_{\mathrm{d}}} \exp \left(-\frac{\left(t-t^{\prime}\right) p^{2}}{\tau_{\mathrm{d}}}\right)
$$

$\bar{e}$ is the function given by $(6.5)$ and $\langle\ldots\rangle^{\prime}$ an average with respect to the creation distribution function $\hat{\psi}$. Note that the quantity $\langle\bar{e} \bar{e}\rangle$ ' is a 'universal tensor', fully determined by the macroscopic deformation gradient $\boldsymbol{F}_{t}\left(t^{\prime}\right)$ and independent of material properties.

Recently a modified tube model has been proposed by Jongschaap (1988). This model, called the reptating rope model, was intended to clarify some discrepancies between the Doi and Edwards model, described above, and the Curtiss-Bird model, which will be discussed in the next section. The model differs from the Doi and Edwards model in the sense that the primitive chain is no longer treated as the average of a Gaussian chain in which the internal stresses are determined by entropic forces, but as an inextensible rope in which the tensile stresses are determined by the external forces. The tube is of about the same (unspecified) diameter as the rope. Two kinds of forces are acting upon the rope: Brownian forces, due to the one-dimensional Brownian motion of the rope in the tube (the ends of the tube are acting as absorbing barriers), and hydrodynamic forces, due to the friction between the rope and the tube wall. The latter occurs because of the difference $(u-v)$ in velocity between the inextensible rope (so $\partial a / \partial s=0$ ) and the affinely moving walls of the tube (so $\partial v / \partial s=$ $\boldsymbol{e} \cdot \boldsymbol{D} \cdot \boldsymbol{e})$. The resulting stress per segment due to both effects was proved to be 
$\bar{\sigma}=\bar{\sigma}^{\mathrm{B}}+\bar{\sigma}^{\mathrm{H}}$ with

$$
\bar{\sigma}^{\mathrm{B}}=\frac{k T}{L}
$$

due to Brownian forces and

$$
\bar{\sigma}^{\mathrm{H}}=\zeta \int_{0}^{s} \int_{\frac{1}{2} L}^{s^{\prime \prime}} e\left(s^{\prime}, t\right) \cdot \boldsymbol{D} \cdot \boldsymbol{e}\left(s^{\prime}, t\right) \mathrm{d} s^{\prime} \mathrm{d} s^{\prime \prime}
$$

in which $\zeta$ is a frictional coefficient, due to frictional forces.

The derivation of a constitutive equation is similar to the one described above in connection with the Doi and Edwards equation (6.11), if in addition one assumes that

$$
\left\langle\boldsymbol{e}\left(s^{\prime}, t\right) \boldsymbol{e}\left(s^{\prime}, t\right) \boldsymbol{e}(s, t) \boldsymbol{e}(s, t)\right\rangle \simeq\langle\boldsymbol{e}(s, t) \boldsymbol{e}(s, t) \boldsymbol{e}(s, t) \boldsymbol{e}(s, t)\rangle .
$$

This means that the rope should be mildly curved in some sense. A similar assumption of mild curvature is also made in the theory of Curtiss and Bird, to be described in the next section.

The constitutive equation of the reptating rope model finally becomes

$$
T_{p}=n N k T\left(\int_{-\infty}^{t} \mu\left(t-t^{\prime}\right)\left\langle\langle\bar{e} \bar{e}\rangle^{\prime} \mathrm{d} t^{\prime}+\int_{-\infty}^{t} \phi\left(t-t^{\prime}\right) D:\langle\bar{e} \bar{e} \bar{e} \bar{e}\rangle^{\prime} \mathrm{d} t^{\prime}\right)\right.
$$

in which

$$
\phi\left(t-t^{\prime}\right)=\frac{\zeta}{2 k T} \int_{0}^{L} s(L-s) \chi\left(t-t^{\prime}, s\right) \mathrm{d} s .
$$

By inserting $\chi=-(\partial / \partial t) \Phi$, using the diffusion equation (6.1) and integrating by parts one obtains $\phi\left(t-t^{\prime}\right)=1 / L \int_{0}^{L} \Phi\left(t-t^{\prime}, s\right) \mathrm{d} s$. If we compare this with (6.12) we see that the functions $\phi$ and $\mu$ are related as

$$
\mu=-\frac{\partial \phi}{\partial t} .
$$

The assumption that the rope is inextensible may be justified by the fact that in relatively slow flows the length of the primitive chain (in the Doi and Edwards sense) is not expected to change very much. In flows with fast and sudden changes in the flow field such will be not the case. Then, instead of an inextensible rope an elastic rope is expected to be more appropriate. A theory of this kind has recently been worked out by Geurts and Jongschaap (1988). In that paper the mild curvature assumption (6.15) has also been reconsidered.

In our brief introduction to the tube model we have concentrated on some basic principles and left out of consideration a lot of interesting topics which are still the subject of current research. Among these we have the phenomena of contour length fluctuations and of tube reorganisation. The latter is especially important in branched polymers, in linear polymers with polydispersity and in mixtures of different polymers. For an introduction to this field we refer to the book by Doi and Edwards (1986).

\subsection{The Curtiss-Bird model}

In the theory of Curtiss and Bird (1981) no tube was used in order to obtain a reptation motion. Instead, a molecule was represented by a Kramers chain, consisting of $N$ 
beads and $N-1$ rods, each of length $a$ and with orientation (unit) vectors $e_{i}$. The reptation motion occurs since the friction forces on the beads are given by an anisotropic Stokes' law. In particular, for the difference between the frictional forces on two subsequent beads, one assumes

$$
f_{i+1}^{\mathrm{H}}-f_{i}^{\mathrm{H}}=-N^{\gamma} a \zeta_{i} \cdot\left(\dot{e}_{i}-L \cdot e_{i}\right)
$$

Here, $\gamma$ is a model parameter, called the chain constraint exponent and $\zeta_{i}$ is a friction tensor, given by

$$
\zeta_{i}=a \zeta\left[1-(1-\varepsilon) e_{i} e_{i}\right]
$$

The parameter $\varepsilon$, called the link tension coefficient, determines the anisotropy of the friction. For $\varepsilon=0$ we have $\left(f_{i+1}^{\mathrm{H}}-f_{i}^{\mathrm{H}}\right) \cdot e_{i}=0$ which means that the tension in all links becomes zero, for $\varepsilon \neq 0$ the tension can vary from point to point. In (6.20) we have added a factor $a$ in order to give $\zeta$ the meaning of friction per unit length, like we did in the preceding section. Next, in order to derive a diffusion equation on the basis of their phase-space kinetic theory Curtiss and Bird introduced the so-called 'reptation approximation'. This statement about the anisotropy of the Maxwellian velocity distribution reads

$$
\overline{\left(u_{i}-v\right)\left(u_{j}-v\right)}=\frac{k T}{2 m}\left(e_{i-1}+e_{i}\right)\left(e_{j-1}+e_{j}\right) .
$$

Here $\boldsymbol{u}_{i}-\boldsymbol{v}$ is the velocity of bead $i$, with respect to the macroscopic velocity $\boldsymbol{v}$, and the overbar denotes an average with respect to the velocity distribution. The idea is that on the average the relation velocity $\boldsymbol{u}_{i}-\boldsymbol{v}$ of bead $i$ should have approximately the same direction as the average of the directions of the two links associated with the bead. The next assumption needed in the theory is the 'mild curvature assumption'. This means that orientations of adjacent links are assumed not to vary significantly. This assumption is similar to the one stated in equation (6.15) in the reptating rope model.

On the basis of these assumptions Curtiss and Bird were able to derive an evolution (diffusion) equation for the single-link distribution function $\psi(e, s, t)$, in which $s=i L / N$ is a continuous variable equivalent to the bead number $i$. After a lengthy calculation using the phase-space formalism an equation of the same shape as equation (6.8) of the Doi and Edwards theory was obtained. The only difference is that the diffusion constant $D$ according to Curtiss and Bird differs by a factor $2 / N^{\gamma}$ from the one of Doi and Edwards. This difference is not very serious inasmuch as it enters in particular in the time constant $\tau_{d}(6.4)$ which is to be determined empirically.

The solution of the diffusion equation is stated again in the form (6.3) and this is used to perform the averaging in the calculation of the stress tensor. To this end Curtiss and Bird use an expression similar to our expression (3.12). On using again the generalised Stokes' law (6.19) for the frictional force, the mild curvature approximation and the diffusion equation they obtain

$$
T_{p}=n N^{1+\gamma} \zeta \int_{0}^{L} s(L-s)\left(\frac{\delta}{\delta t}\langle e e\rangle-2(1-\varepsilon) \boldsymbol{D}:\langle\text { eeee }\rangle\right) \mathrm{d} s
$$

in which $\delta / \delta t$ is the upper convected derivative (A2.12). This expression is of the same type as the Giesekus expression (3.15) discussed earlier. From the diffusion 
equation (6.8) it can be proved that

$$
\frac{\delta}{\delta t}\langle e e\rangle=\frac{2 D}{N^{\gamma}}\langle e e\rangle-2 D:\langle e e e e\rangle .
$$

The diffusion coefficient $D$ is given by (6.2). If the expression (6.23) is introduced in (6.22) and the averages are transformed into averages with respect to $\hat{\psi}\left(e^{\prime}\right)$ by using the integral expression (6.7) of $\psi(e, t, s)$ one finally obtains

$$
T_{p}=n N k T\left(\int_{-\infty}^{t} \mu\left(t-t^{\prime}\right)\langle e e\rangle^{\prime} \mathrm{d} t^{\prime}+2 \varepsilon \int_{-\infty}^{t} \phi\left(t-t^{\prime}\right) D:\langle e e e e\rangle^{\prime} \mathrm{d} t^{\prime}\right)
$$

in which $\mu$ and $\phi$ are the same functions as used in equation (6.16). In fact, we see that the results (6.24) and (6.16) are identical, up to a factor $2 \varepsilon$ in the last term (for $\varepsilon=0.5$ both results coincide) .

In our brief review we have had no opportunity to discuss the details of the Curtiss-Bird theory. To this end we refer to the original papers and the textbook by Bird et al (1987b). One of the main features of this approach, however, still should be mentioned. That is that it proves that the results of a tube model may also be derived in a phase-space kinetic theory based upon a kind of bead-rod-spring model and closely patterned after the dilute solution theories. In this way the possibility to circumvent the more or less artificial tube concept has been proved. On the other hand, it should be noted that the mathematical formulation of the tube models is far simpler than that of the Curtiss-Bird theory.

\section{Generalised continuum theories}

\subsection{Introduction}

In $\S 2$ we have seen that in the continuum approach it is possible to obtain results on the basis of a few general principles. In this way, however, no use is made of information about the microstructure of the materials. In order to retain the advantages of the continuum theory but still be able to include certain aspects of the microstructure various generalised continuum theories have been proposed. Well known examples are the theory of Ericksen (1960) in which with each material point in the continuum is associated a single preferred direction, the theory of Hand (1962) in which with each material point of the continuum is associated a second-order tensor, the theory of 'microfluids' by Eringen (1964) and theories based upon the Cosserat continuum (Cosserat and Cosserat 1907) in which each material point of the continuum has the geometric properties of a rigid body. We will not attempt to give a survey of these approaches here, but instead concentrate upon a recent theory by Grmela (1986a) which was shown to be especially relevant to polymeric systems (Grmela 1986a, b, Ait-Kadi 1988, Ajji et al 1989) and general enough to include some important earlier theories. We will follow here a treatment, presented recently (Jongschaap 1989), in which a new derivation and an extension of this theory was given.

\subsection{Configuration tensor models}

During the flow of a material a part of the energy supply is stored and a part of it is dissipated. Under isothermal conditions the storage is described by the free-energy 
functional $A\{\Phi\}$ in which $\Phi$ is a state variable, characterising the internal (macromolecular) structure of the fluid. In general $\Phi$ may be a function, e.g. a configurational distribution function. In this review we will restrict ourselves to the case that $\Phi$ is a symmetric second-order tensor $S$, the so-called configuration tensor. So we have $A=A(S)$.

The rate of energy dissipation $\sigma$ is the difference between the power input and the storage, so

$$
\sigma=T: L-\dot{A}=T: L-\frac{\partial A}{\partial S}: \dot{S}
$$

We now consider the transformation $L \rightarrow-L$, i.e. a 'macroscopic time reversal'. Denoting variables which are even or odd under this transformation with an upper index '+' or '-' respectively, we have: $\sigma=\sigma^{+}$since by the second law of thermodynamics the dissipation should be positive, irrespective of the sign of $L ; \partial A / \partial S=(\partial A / \partial S)^{+}$ since $A$ and $S$ are state variables and $\boldsymbol{L}=\boldsymbol{L}^{-}$, by definition.

By taking the even part of (7.1) we obtain for the dissipation

$$
\sigma=T^{-}: L-\frac{\partial A}{\partial S}: \dot{S}^{+}
$$

From the parity of the variables and the inequality $\sigma \geqslant 0$ it follows that the only admissible couplings among the variables in this expression are

$$
T^{-}=f(L) \quad \dot{S}^{+}=g\left(\frac{\partial A}{\partial S}\right) .
$$

So, each term in (7.2) is purely dissipative. Therefore we define dissipative and reversible parts of $\boldsymbol{T}$ and $\dot{\boldsymbol{S}}$ as

$$
\begin{array}{ll}
T^{\mathrm{D}}=T^{-} & \dot{S}^{\mathrm{D}}=\dot{S}^{+} \\
T^{\mathrm{R}}=T^{+} & \dot{S}^{\mathrm{R}}=\dot{S}^{-} .
\end{array}
$$

We now take the odd part of equation (7.1):

$$
T^{\mathrm{R}}: L-\frac{\partial A}{\partial \boldsymbol{S}}: \dot{\boldsymbol{S}}^{\mathrm{R}}=0 .
$$

From the parity of the variables it now follows that the only admissible couplings are

$$
\boldsymbol{T}^{\mathrm{R}}=k\left(\frac{\partial A}{\partial \boldsymbol{S}}\right) \quad \dot{\boldsymbol{S}}^{\mathrm{R}}=\boldsymbol{m}(\boldsymbol{L}) .
$$

By differentiation of (7.5) with respect to $L$ and using (7.6) we obtain

$$
\boldsymbol{T}^{\mathrm{R}}=\frac{\partial A}{\partial \boldsymbol{S}}: \frac{\partial \dot{\boldsymbol{S}}^{\mathrm{R}}}{\partial \boldsymbol{L}} .
$$

This is a special case of the general expression for the stress tensor (3.38), first derived by Grmela (1985). The derivation presented here is based upon a method by Martin et al (1972).

By introducing the generalised thermodynamic force $M \equiv \partial A / \partial S$ and the fourthorder tensor $\boldsymbol{\Lambda} \equiv \partial \dot{\boldsymbol{S}}^{\mathrm{R}} / \partial \boldsymbol{L}$ we rewrite (7.7) as

$$
\boldsymbol{T}^{\mathrm{R}}=\boldsymbol{M}: \Lambda=\Lambda^{\mathrm{T}}: M
$$


in which the transposed fourth-order tensor $\Lambda$ is defined by $\Lambda_{i j k m}^{\mathrm{T}}=\Lambda_{k m i j}$. In the second equation (7.6) we will consider the case that the function $m$ is linear in $L$, so

$$
\dot{\boldsymbol{S}}^{\mathrm{R}}=\boldsymbol{\Lambda}: \boldsymbol{L} \text {. }
$$

If, also, the equations (7.3) are rewritten as

$$
T^{\mathrm{D}}=\boldsymbol{\eta}: L \quad \dot{S}^{\mathrm{D}}=-\boldsymbol{\beta}: \boldsymbol{M}
$$

in which $\boldsymbol{\eta}$ and $\beta$ are fourth-order tensors, the results (7.8), (7.9) and (7.10) can be summarised in matrix form:

$$
\left(\begin{array}{c}
T \\
\dot{S}
\end{array}\right)=\left(\begin{array}{cc}
\eta & -\Lambda^{\mathrm{T}} \\
\boldsymbol{\Lambda} & \boldsymbol{\beta}
\end{array}\right):\left(\begin{array}{c}
L \\
-\boldsymbol{M}
\end{array}\right)
$$

Similar expressions have been obtained in a different way by Martin et al (1972), Leonov (1976), Lhuillier and Ouibrahim (1980), Stickforth $(1981,1986)$ and others. The skew symmetry of the matrix in (7.11) is in accordance with Onsager-Casimir reciprocal relations (Onsager 1931, 1932, Casimir 1945) for the rate equations based upon the expression (7.1) of the dissipation. The formulation of specific forms of the present theory consists of a specification of tensors $\boldsymbol{\eta}, \boldsymbol{\Lambda}$ and $\boldsymbol{\beta}$ and the function $\boldsymbol{M}=\boldsymbol{M}(\boldsymbol{S})$.

\subsection{Applications}

As a first example we discuss a model by Giesekus $(1966,1982)$. In this model there is reversible stress of the neo-Hookean type

$$
T^{\mathrm{R}}=\mu(S-1)
$$

and rate equation for $S$

$$
\stackrel{\nabla}{\boldsymbol{S}}=-\boldsymbol{B} \cdot \boldsymbol{T}^{\mathrm{R}}
$$

in which $B=\eta^{-1}\left(1+a T^{\mathrm{R}}\right)$ is a generalised mobility tensor.

From (7.12) and (7.13) a constitutive equation of the type (2.59) is obtained. On the other hand it can be shown that the Giesekus model is consistent with the matrix formulation (7.11) if we define

$$
\begin{aligned}
& \Lambda_{i j k m}=\delta_{i k} S_{m j}+S_{i m} \delta_{k j} \\
& \boldsymbol{M}=\frac{1}{2} \mu\left(\mathbf{1}-\boldsymbol{S}^{-1}\right) \\
& \boldsymbol{\beta}=\boldsymbol{B} \cdot \boldsymbol{\Lambda} .
\end{aligned}
$$

As a second example we consider the Leonov model (Leonov 1976). To this end, on using (7.10) the expression (7.11) is rewritten in the form

$$
\left(\begin{array}{l}
T^{\mathrm{D}} \\
L^{\mathrm{D}}
\end{array}\right)=\left(\begin{array}{ll}
\boldsymbol{\eta} & 0 \\
\mathbf{0} & \nu
\end{array}\right):\left(\begin{array}{c}
\boldsymbol{L} \\
-T^{\mathrm{R}}
\end{array}\right) .
$$

The tensors $L^{\mathrm{D}}$ and $\boldsymbol{\nu}$ are given by $\boldsymbol{L}^{\mathrm{D}}=-\boldsymbol{\Lambda}^{-1}: \dot{S}^{\mathrm{D}}$ and $\boldsymbol{\nu}=\boldsymbol{\Lambda}^{-1}: \boldsymbol{\beta}: \boldsymbol{\Lambda}^{-\mathrm{T}}$. The result (7.15) is similar to an expression proposed by Leonov. There is, however, an important difference, namely that the off-diagonal elements in (7.15) are zero, whereas in the Leonov model they may be non-zero. This difference is a consequence of the different ways of defining the reversible and dissipative parts of $\boldsymbol{T}$ and $\boldsymbol{L}$. In our approach this 
was based upon a distinction between odd and even variables with respect to 'macroscopic time reversal' while in the theory of Leonov it is based upon the concept of an elastic (recoverable) strain. A similar situation arises in theories based upon Eckart's concept of a 'variable relaxed state' (Eckart 1948, Stickforth 1986).

The applicability of the matrix formulation (7.11) is not restricted to generalised continuum models but may be applied to microscopic models as well. To this end the relevant thermodynamic variables have to be determined. In a configuration space description, for instance, we would start with the free energy as a functional of the distribution function: $A=A\{\dot{\psi}\}$ and at the level of a description with a structure tensor, like $S=\langle q q\rangle$, with the free energy as a function of $S: A=A(S)$, as has already been done above. Often, however, it turns out to be useful to express the free energy as a function of the configuration itself. We will illustrate this for some micro-rheological models.

First, consider the elastic dumbbell model ( $\$ 4.2)$. In that case, the thermodynamic force becomes

$$
\boldsymbol{m}=-\boldsymbol{f}^{\mathrm{B}}+\boldsymbol{f}^{\mathrm{1}}=k T \frac{\partial}{\partial \boldsymbol{q}} \ln \frac{\psi}{\psi_{0}} .
$$

The associated free energy $a=k T \ln \psi / \psi_{0}$ may be considered as the local value in configuration space of the free-energy functional

$$
A\{\psi\}=k T \int \psi \ln \frac{\psi}{\psi_{0}} \mathrm{~d}^{3} q
$$

which (within an additive constant) is the so called 'dynamical free energy', introduced by Doi (1983). By (3.19) the particle contribution to the stress tensor may be written as $T_{p}=n\langle\tau\rangle$ in which

$$
\tau=m q
$$

In order to obtain a matrix form of the type (7.11), we note that since $\tau: L=\boldsymbol{m q}: \boldsymbol{L}=$ $\boldsymbol{m} \cdot \boldsymbol{L} \cdot \boldsymbol{q}=\boldsymbol{m} \cdot\left(\boldsymbol{v}_{2}-\boldsymbol{v}_{1}\right)$ is the total energy supplied to the dumbbell and $\dot{a}=(\partial a / \partial \boldsymbol{q}) \cdot \dot{\boldsymbol{q}}=$ $\boldsymbol{m} \cdot \dot{q}$ is the storage of energy, the dissipation becomes

$$
\sigma=\boldsymbol{\tau}: \boldsymbol{L}-\boldsymbol{m} \cdot \dot{q} \text {. }
$$

Analogous to the derivation of (7.11) from (7.1) a similar expression may be obtained from (7.19). In the case of the dumbbell model we may derive a more specific result, using the expression (7.18) for $\tau$ and the equation of motion (4.2)

$$
\dot{q}=L \cdot q-\frac{2}{\zeta} m
$$

So, the matrix equation, corresponding to the dissipation form (7.19) becomes

$$
\left(\begin{array}{l}
\tau \\
\dot{q}
\end{array}\right)=\left(\begin{array}{cc}
0: & -(1 q)^{\mathrm{T}} \cdot \\
1 q: & 2 / \zeta 1 \cdot
\end{array}\right)\left(\begin{array}{c}
L \\
-m
\end{array}\right) .
$$

Here, the transpose of a third-order tensor is defined as $a_{i j k}^{\mathrm{T}}=a_{i k j}$.

In the case of a rigid dumbbell, by equation (3.24) we have: $T=n\left\langle\tau^{R}\right\rangle+n\left\langle\tau^{D}\right\rangle$ with $\tau^{\mathrm{R}}=-\boldsymbol{P} \cdot f^{\mathrm{B}} q$ and $\tau^{\mathrm{D}}=(1-P) \cdot f^{\mathrm{H}} q$. If again we define $\boldsymbol{m}=-f^{\mathrm{B}}+f^{\mathrm{l}}$, we obtain $\boldsymbol{\tau}^{\mathrm{R}}=$ $-\boldsymbol{P} \cdot \boldsymbol{m} \boldsymbol{q}$, since in this case $\boldsymbol{P} \cdot f^{1}=\mathbf{0}$. On the other hand, by substitution of the expression 
for $f^{H}$ analogous to (3.14) we obtain: $\tau^{\mathrm{D}}=\frac{1}{2} \zeta q^{2}$ eeee $: D=\frac{1}{2} \zeta q^{2}(1-P) e e: L$. These expressions, collected in a matrix form similar to (7.11), become

$$
\left(\begin{array}{l}
\boldsymbol{\tau} \\
\dot{q}
\end{array}\right)=\left(\begin{array}{cc}
\frac{1}{2} \zeta q^{2}(1-P) e e: & -(\boldsymbol{P q})^{\mathrm{T}} \\
\boldsymbol{P q}: & (2 / \zeta) P \cdot
\end{array}\right)\left(\begin{array}{c}
\boldsymbol{L} \\
-\boldsymbol{m}
\end{array}\right) .
$$

As we see, the expressions (7.21) and (7.22) are very similar. The main difference is the diagonal element corresponding to $\tau^{\mathrm{D}}$ and the occurrence of the projection operator $P$ in (7.22).

As the next example of application we consider the reptation models, discussed in $\S 6$. In the case of the Doi and Edwards model we have to deal with two motions: the change of orientation of the $e$ vectors and a one-dimensional diffusion along the rope. The first process is determined by the equation of motion (6.6) which may be written as

$$
\dot{e}=(1-e e) e: L
$$

and the second process by the diffusion law

$$
\dot{s}=-D \frac{\partial \ln \psi}{\partial s} \text {. }
$$

We now introduce two thermodynamic forces. First, the force

$$
\boldsymbol{m}=k T \frac{\partial \ln \psi}{\partial \boldsymbol{e}}
$$

associated with the motion of the $e$ vectors and

$$
\mu=k T \frac{\partial \ln \psi}{\partial s}
$$

associated with the motion along the tube. We will show now that the stress tensor is given by $\boldsymbol{T}=\left(n N / L \int_{0}^{L}\langle\boldsymbol{\tau}\rangle \mathrm{d} s\right.$ in which (similar to (7.23))

$$
\tau=[(1-e e) e]^{\mathrm{T}} \cdot \boldsymbol{m} \text {. }
$$

This will be done by showing that (7.27) is in accordance with the Doi and Edwards result (6.11). To this end, first note that since $e \cdot \partial / \partial e=0$ it follows from (7.25) that (7.27) reduces to $\tau=m e$. Furthermore, in this expression, by (7.25) and (6.7) we have

$$
m=\frac{k T}{\psi} \int_{-\infty}^{t} \int \chi\left(t-t^{\prime}, s\right) \frac{\partial}{\partial \boldsymbol{e}} \delta(e-\bar{e}) \hat{\psi}\left(e^{\prime}\right) \mathrm{d}^{2} e^{\prime} \mathrm{d} t^{\prime} .
$$

So, the stress tensor becomes

$$
\boldsymbol{T}=\frac{n k T}{L} \int_{-\infty}^{t} \int_{0}^{L} \iint \chi\left(t-t^{\prime}, s\right)\left(\frac{\partial}{\partial \boldsymbol{e}} \delta(\boldsymbol{e}-\bar{e})\right) \hat{e} \hat{\psi}\left(\boldsymbol{e}^{\prime}\right) \mathrm{d}^{2} \boldsymbol{e}^{\prime} \mathrm{d}^{2} \boldsymbol{e} \mathrm{d} s \mathrm{~d} t^{\prime}
$$

By performing an integration by parts with respect to $e$ (note that

$$
\left.\int\left(\frac{\partial}{\partial e} \phi\right) e \mathrm{~d}^{2} e=\int(3 e e-1) \phi \mathrm{d}^{2} e\right)
$$

and an integration with respect to $e^{\prime}$ and $s$ we obtain

$$
T=-n k T 1+3 n N k T \int_{-\infty}^{t} \mu\left(t-t^{\prime}\right)\langle\overline{\boldsymbol{e}} \overline{\boldsymbol{e}}\rangle^{\prime} \mathrm{d} t^{\prime}
$$


This result differs only by an isotropic term from the Doi and Edward result (6.11). This proves the expression (7.27) for $\tau$.

So we see that also for the Doi and Edwards theory it is possible to give a matrix formulation similar to our general equation (7.11). In this case we have

$$
\left(\begin{array}{c}
\tau \\
\dot{e} \\
\dot{s}
\end{array}\right)=\left(\begin{array}{ccc}
0: & -[(1-e e) e]^{\mathrm{T}} . & 0 \\
(1-e e) e: & 0 \cdot & 0 \\
0: & 0 . & D / k T
\end{array}\right)\left(\begin{array}{c}
L \\
-m \\
-\mu
\end{array}\right) .
$$

In the case of the reptating rope model an additional viscous stress comes into play. From (6.16) and (6.17) we see that this corresponds to a contribution $\tau^{D}=$ $\frac{1}{2} \zeta s(L-s)$ eeee $: L$ to $\tau$. As a result, the matrix representation $(7.31)$ is changed into

$$
\left(\begin{array}{c}
\boldsymbol{\tau} \\
\dot{e} \\
\dot{s}
\end{array}\right)=\left(\begin{array}{ccc}
\frac{1}{2} \zeta s(L-s) \text { eeee }: & -[(\mathbf{1}-e e) e]^{\mathrm{T}} . & 0 \\
(1-e e) e: & 0 . & 0 \\
0: & 0 \cdot & D / k T
\end{array}\right)\left(\begin{array}{c}
L \\
-\boldsymbol{m} \\
-\mu
\end{array}\right) .
$$

An important feature of the present formulation is the skew symmetry of the matrix. This implies a connection between the stress tensor expression and the equations of motion. An interesting example is the case of non-affine motion in a rubber-like network. In a permanent network the stress becomes $T=n\langle\tau\rangle$ with

$$
\tau=m q
$$

in which the thermodynamic force $m=\partial a / \partial q$ is the spring force and $q$ the vector of a segment in a network. In the case of affine motion we have

$$
\dot{q}=\boldsymbol{L} \cdot \boldsymbol{q} .
$$

Formulated in matrix form the expressions (7.23) and (7.34) become

$$
\left(\begin{array}{c}
\tau \\
\dot{q}
\end{array}\right)=\left(\begin{array}{cc}
0: & -\Lambda^{\mathrm{T}} \cdot \\
\Lambda: & 0
\end{array}\right)\left(\begin{array}{c}
L \\
m
\end{array}\right)
$$

with

$$
\Lambda=1 q \text {. }
$$

If we now assume slip in the motion of the segments, for instance slip in entanglements, instead of (7.33) we may have a non-affine convection of the type proposed in the Phan-Thien and Tanner (1977) model (equations (5.25) and (5.26)). In that case we have $\dot{\boldsymbol{q}}=\tilde{\boldsymbol{\Lambda}}: \boldsymbol{L}$ with

$$
\tilde{\Lambda}=\left(1-\frac{\xi}{2}\right) 1 q-\frac{\xi}{2}(1 q)^{\mathrm{T}}
$$

The corresponding matrix expression becomes

$$
\left(\begin{array}{c}
\tau \\
\dot{q}
\end{array}\right)=\left(\begin{array}{cc}
0: & -\tilde{\Lambda}^{T} \cdot \\
\tilde{\Lambda}: & 0 \cdot
\end{array}\right)\left(\begin{array}{c}
L \\
-m
\end{array}\right) .
$$

From (7.35) and (7.38) we see that in the case of non-affine motion not only the equation of motion, but also the stress tensor expression should be modified. This 
point has been discussed in another context by Larson (1981) and also by Maugin and Drouot (1983) and by Grmela (1985). In the present case we have

$$
\begin{aligned}
\boldsymbol{T} & =\langle\boldsymbol{\tau}\rangle=\left\langle\tilde{\boldsymbol{\Lambda}}^{\top} \cdot \boldsymbol{m}\right\rangle \\
& =\left(1-\frac{\xi}{2}\right)\left\langle(\mathbf{1} \boldsymbol{q})^{\mathrm{T}} \cdot \boldsymbol{m}\right\rangle-\frac{\xi}{2}\langle\mathbf{1} \boldsymbol{q} \cdot \boldsymbol{m}\rangle \\
& =\left(1-\frac{\xi}{2}\right)\langle\boldsymbol{m} \boldsymbol{q}\rangle+\text { (isotropic term). }
\end{aligned}
$$

So far we have mainly reformulated a number of macroscopic theories in the framework of a particular type of generalised continuum theory. It should be stressed, however, that just the capability of such a coupling between the continuum approach and the micro-rheological models makes the present formalism useful. On one hand-as has been done in the examples-one may use information from microscopical theories in order to obtain explicit expressions for the matrix elements in (7.11) and for the free-energy function. On the other hand one may modify the microscopical theories, simply by making changes in the matrix elements or in the free-energy function. This is essentially what we did in the example on non-affine motion. In the latter case an essential condition upon the possible changes is the required skew symmetry of the matrix in (7.11).

\section{Discussion}

After an introduction to continuum mechanics and basic elements of micro-rheological modelling we have discussed four important classes of models: bead-rod-spring, transient network, reptation and configuration tensor models. Although it is evident that there are big differences between these models it is important to recognise the common structure in all of them. In each type of model a particular structure is defined with an equation, describing the evolution of it in time, and also an expression relating the stresses and forces in that structure to the macroscopic stress tensor. The evolution equation may consist of a convective, a diffusive and a kinetic part (in configuration tensor models we distinguished a reversible and a dissipative part) and the stress tensor of a reversible and a dissipative part. It is interesting to reconsider the various models from this point of view.

In the bead-rod-spring models we have seen that the occurrence of dissipative stresses is directly related to the presence of constraints, i.e. rigid rods. Furthermore, the evolution equations do not contain kinetic terms. At this point the theory may be extended by including association and dissociation of bead-rod-spring structures or, more generally, time-dependent structures. In the latter case the Rouse matrix (4.18) or its equivalent will become time dependent.

In the transient network theories the evolution equation has a convective and a kinetic part. A diffusive contribution may, however, be included if the 'loss' of segments is considered as a transition from a 'fixed' (network) to a 'free' state in which they act like elastic dumbbeils. A theory of this kind has been worked out by Jongschaap et al (1983) and was generalised recently in the context of a configurational tensor model by Ajji et al (1989).

In our treatment of reptation theories we have seen that the stress tensor is purely reversible in the Doi and Edwards theory. We have also seen how frictional forces 
were included in the reptating rope model and that in that case-as in the Curtiss-Bird model-the stress tensor contains a reversible and a dissipative part. This also became clear in the matrix representations (7.31) and (7.32) in our treatment of reptation in the configuration tensor models. The evolution equation of the tube models contains a convective part, associated with the orientation of tube elements and a diffusive part associated with the motion of the chain along the tube. It is interesting therefore to consider the possibility of kinetic contributions.

First we note that the process of creation and loss of tube elements is similar to the creation and loss of segments in transient-network models. It is indeed possible (Jongschaap and Kamphuis 1986) to formulate the Doi and Edwards theory analogous to the transient-network models. The main difference is that in that case the kinetics is no longer determined by simple rate equations of the type (5.11) but by a diffusion process.

It is also possible to extend the reptation model by introducing additional kinetic processes. This could be done, for instance, by introducing the possibility of adherence and loss of parts of the chain to the tube wall. In this way, a kind of combined reptation and transient-network theory will result.

The convective and diffusive motion in the Curtiss-Bird theory is more complicated than in the tube models since it is based upon the motion of a Kramers chain in a viscous medium. It might be of interest to consider similar models with other types of chain. A possibility close to the reptating rope model, discussed in $\S 6$, would be a worm-like chain with a prescribed flexibility and an anisotropic friction in a viscous medium.

In the configuration tensor approach a clear distinction was made between reversible and dissipative parts of stress and of the evolution equations. This was illustrated in applications to some existing models. In these applications only convective and diffusive but no kinetic processes were discussed. It may be shown, however, that the theory is also applicable to transient-network theories. In that case, the structure tensor $\boldsymbol{S}$ as well as the number density of segments $n$ should be included as a state variable.

being capable of including many of the existing microscopical models, the configuration tensor approach, presented here, may be an attractive tool in formulating modifications of these models. The approach is also very flexible. In this paper only scalars and second-order tensors have been employed as state variables. In some cases it might be necessary to use higher-dimensional or higher-order tensors to represent the microstructure, for instance if one wants to describe the state of a Rouse chain, or the state of a liquid crystal. As already mentioned in $\S 7$ it is also possible to use configurational distribution functions as state variables. In this way a given system may be treated at different levels of description in a unified formalism (see also Grmela 1986a) and also the microscopic theories, based upon a configuration-space description may be reformulated in a compact and transparent way. As we have seen already, this offers the possibility of modifying the theories without entering into the details of the underlying microscopical considerations, such as the averaging procedures (see $\S 3$ ) or the derivation of the evolution equation.

We close this section with a few remarks about the levels of description in the various models. As we have seen, each model may be considered as a simplified representation of a real material. Depending upon the detail of such a representation we speak of a certain level of description. Let us say that at a low level the model structure is very close to reality and that at a high level of description the model structure is a very simplified representation. 
The lowest level of description that has been considered in the present paper was a level at which the structures were described by distributions in configuration space. This means that we did not consider the behaviour of individual particles, but rather the evolution of distribution functions and related quantities. One level of description lower would be a description in phase space. This description has been explored extensively by Bird and co-workers (see Bird et al 1987b). An advantage is that less a priori assumptions have to be made in deriving the basic stress tensor and evolution expressions. On the other hand the formalism becomes more complicated.

The next level of description is one in which not the probability densities but the individual particles are considered. This level has received little attention in the past, since an overwhelming number of coordinates is needed to describe a system in this way. Even a mean-field approach, in which the stochastic motion of only one particle is considered seems to be tractable only in the simplest cases. Recently, however, this situation has been changed, since powerful computers are available, by which simulations of various systems at this level of description become possible. An advantage of this method is that model structures which are too complex for an anaytical treatment may often still be used in numerical simulations.

It is good to realise that the simple fluid theory discussed in $\S 2.2$ may also be considered as a model with a high level of description. At this level no information about the microstructure is retained, but, as we have seen, by introducing some plausible axioms it is possible to obtain a useful classification of constitutive equations and special types of flow. The configuration tensor approach discussed in $\S 7$ uses a level of description inbetween the simple fluid theory and the microscopical models.

Which level of description is useful for a given material in a particular situation depends upon its purpose. In the context of flow problems a high level of description is often the most appropriate while a study of the influence of structural changes in materials on their flow behaviour requires a lower level of description. In any case one should try to achieve an optimum between mathematical simplicity, physical reality and usefulness.

\section{Appendix 1. Frames of reference}

The points, vectors and tensors, used in rheological models may be considered as the images of the corresponding physical quantities under a mapping from the real physical space to a three-dimensional Euclidean space. We may imagine this mapping to be the registration of a process by an observer. If one process is registered by different observers with different positions and orientations and with a relative motion with respect to each other their images of the same process will be different. The transformation between such images is called a change of frame (Noll 1958, Truesdell and Noll 1965). A moving point, described by $\boldsymbol{x}(t)$ in one frame becomes in a second frame

$$
x^{*}(t)=c(t)+Q(t) \cdot\left(x-x_{0}\right)
$$

Here $c(t)$ is a moving point, $Q(t)$ an orthogonal tensor and $x_{0}$ a fixed point.

From (A1.1) it can be seen that the vector $\boldsymbol{u}=\boldsymbol{y}-\boldsymbol{x}$ between two points transforms like $\boldsymbol{u}^{*}=\boldsymbol{Q} \cdot \boldsymbol{u}$. Such types of vector are called objective. An objective tensor is one which transforms like $\boldsymbol{S}^{*}=\boldsymbol{Q} \cdot \boldsymbol{S} \cdot \boldsymbol{Q}^{\top}$. We see that if $\boldsymbol{u}=\boldsymbol{S} \cdot \boldsymbol{v}$ and $\boldsymbol{u}, \boldsymbol{S}$ and $\boldsymbol{v}$ are objective, then $u^{*}=S^{*} \cdot v^{*}$. 
In continuum mechanics one uses objective as well as non-objective tensors. From (A1.1) and the definition (2.2), for instance, it follows that the deformation gradient $F_{i}(\tau)$ transforms like

$$
F_{t}(\tau)^{*}=Q(\tau) \cdot F_{t}(\tau) \cdot Q^{T}(t)
$$

under a change of frame. So this tensor is not objective. On the other hand from the basic expression $t=T \cdot n$ and the objectivity of the vectors $t$ and $n$ it follows that the stress tensor $T$ is objective: $T^{*}=Q \cdot T \cdot Q^{T}$.

For reference purposes we now list the transformation rules of some other important tensors of continuum mechanics. The relative Cauchy tensor (2.4):

$$
C_{i}^{*}(\tau)=Q(t) \cdot C_{t}(\tau) \cdot Q^{T}(t) .
$$

The velocity gradient tensor (2.8):

$$
L^{*}=\boldsymbol{Q} \cdot L \cdot Q^{\mathrm{T}}+\dot{Q} \cdot Q^{\mathrm{T}}
$$

The rate of strain tensor (2.9):

$$
D^{*}=Q \cdot D \cdot Q^{\mathrm{T}}
$$

The vorticity tensor $(2.10)$ :

$$
W^{*}=Q \cdot W \cdot Q^{\mathrm{T}}+\dot{Q} \cdot \boldsymbol{Q}^{\mathrm{T}}
$$

The Rivlin-Ericksen tensors (2.11)

$$
\boldsymbol{A}_{n}^{*}=\boldsymbol{Q} \cdot \boldsymbol{A}_{n} \cdot \boldsymbol{Q}^{\mathrm{T}}
$$

An important application of the concept of a change of frame is the principle of material frame indifference, discussed in $\$ 2.2$. We will illustrate this now for the derivation of equation (2.17).

By the principle of material frame indifference, the constitutive equation (2.15) should obey the equation

$$
\boldsymbol{Q}(t) \cdot \boldsymbol{T}(t) \cdot \boldsymbol{Q}^{\mathrm{T}}(t)=\underset{\tau=-\infty}{\stackrel{t}{\mathscr{F}^{\prime}}}\left\{\boldsymbol{Q}(\tau) \cdot \boldsymbol{F}_{t}(\tau) \cdot \boldsymbol{Q}^{\mathrm{T}}(t)\right\}
$$

for arbitrary orthogonal tensor histories $Q(\tau)(-\infty<\tau<t)$. On using the polar decomposition theorem (2.3), $\boldsymbol{F}_{t}(\tau)$ is written as $\boldsymbol{F}_{t}(\tau)=\boldsymbol{R}_{t}(\tau) \cdot \boldsymbol{U}_{t}(\tau)$. Since $\boldsymbol{Q}(\tau)$ is arbitrary we may take $\boldsymbol{Q}(\tau)=\boldsymbol{R}_{t}^{\mathrm{T}}(\tau)$. Then, $\boldsymbol{Q}(t)=\mathbf{1}$ and $\boldsymbol{Q}(\tau) \cdot \boldsymbol{F}_{\mathrm{r}}(\tau)=\boldsymbol{U}_{\mathrm{r}}(\tau)$, so

$$
T(t)=\underset{\tau=-\infty}{\stackrel{t}{F^{\prime}}}\left\{U_{t}(\tau)\right\}
$$

Since $\boldsymbol{U}_{t}^{2}(\tau)=\boldsymbol{C}_{t}(\tau)$ this expression is equivalent to (2.17). We have now proved that this result is a necessary condition for frame indifference. That it is also sufficient may be proved by inspection.

\section{Appendix 2. Convected derivatives}

In the formulation of constitutive equations instead of the usual local $(\partial / \partial t) \boldsymbol{M}(\boldsymbol{x}, t)$ or the material $\dot{\boldsymbol{M}}=(\mathrm{d} / \mathrm{d} t) \boldsymbol{M}(\boldsymbol{x}(t), t)$ time derivative one often makes use of so-called convected derivatives. These derivatives were first used in the formulation of constitutive equations in a classical paper by Oldroyd (1950). In this paper the presentation is based upon convected coordinates. Here we will give a coordinate-free treatment (see also Bolder 1969). 
Let $\boldsymbol{M}(\tau)$ be the value of a tensor field, associated with a certain particle at time $\tau$, and consider a linear transformation (fourth-order tensor) $\Gamma(t, \tau)$ by which $M(\tau)$ is transformed into $\bar{M}(t, \tau)=\Gamma(t, \tau): M(\tau)$. This transformation-called an entrainment law-is taken to be associative in the sense that

$$
\Gamma(t, \tau)=\Gamma(t, s): \Gamma(s, \tau)
$$

It describes a way of 'dragging along' the tensor field $\boldsymbol{M}$ in a specified manner, related to the flow of the material.

The convective derivative $(\delta / \delta \tau) M$, associated with the entrainment law $\Gamma$ is defined then by the commutative diagram

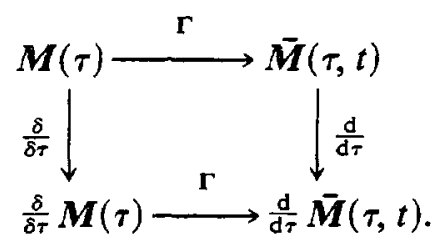

So we have $\Gamma:(\delta / \delta t) \boldsymbol{M}=(\mathrm{d} / \mathrm{d} \tau)(\Gamma: M)$. The quantity $(\delta / \delta \tau) \boldsymbol{M}(\tau)$, defined in this manner, can be proved to be independent of $t$ and we also see that it vanishes if $\bar{M}(t, \tau)$ becomes independent of $\tau$. In that case the change in $\boldsymbol{M}$ with $\tau$ is entirely compensated by the entrainment law $\Gamma$. In general, one could say that $(\delta / \delta \tau) \boldsymbol{M}(\tau)$ measures the change of $\boldsymbol{M}$ with respect to a given entrainment law.

From diagram (A2.2), on performing the differentiation of $\bar{M}(t, \tau)$, the following expression for the convected derivative is obtained

$$
\frac{\delta}{\delta \tau} \boldsymbol{M}=\dot{M}+\boldsymbol{\Lambda}: M
$$

In this expression $\Lambda$ is a fourth-order tensor, given by

$$
\Lambda=\Lambda(\tau)=\Gamma(t, \tau)^{-1}: \frac{\mathrm{d}}{\mathrm{d} \tau} \Gamma(t, \tau)
$$

This tensor may be proved to be independent of the time $t$.

We now consider the case that $\boldsymbol{\Lambda}$ is a function of the velocity gradient tensor $\boldsymbol{L}$. Then we have

$$
\frac{\delta}{\delta \tau} \boldsymbol{M}=\dot{M}+f(D, W, M)
$$

In continuum mechanics it is useful to consider convected derivatives that are objective if $\boldsymbol{M}$ is an objective tensor (see Appendix 1). In that case from (A2.5) the following restriction upon the function $f$ is obtained:

$$
\begin{aligned}
& f(D, W, M)=Q^{\mathrm{T}} \cdot \dot{Q} \cdot \boldsymbol{M}+\boldsymbol{M} \cdot \dot{Q}^{\mathrm{T}} \cdot Q+ \\
& f\left(\boldsymbol{Q} \cdot \boldsymbol{D} \cdot Q^{\mathrm{T}}, \boldsymbol{Q} \cdot \boldsymbol{W} \cdot \boldsymbol{Q}^{\mathrm{T}}+\dot{Q} \cdot \boldsymbol{Q}^{\mathrm{T}}, \boldsymbol{Q} \cdot \boldsymbol{M} \cdot \boldsymbol{Q}^{\mathrm{T}}\right)
\end{aligned}
$$

By taking $Q=1$ and $\dot{Q}=-W$ we obtain $f(D, W, M)=-W \cdot M+M \cdot W+g(D, M)$ with $g(D, M)=f(D, O, M)$. So the convected derivative becomes

$$
\frac{\delta}{\delta \tau} M=\dot{M}-W \cdot M+M \cdot W+g(D, M)
$$


Furthermore, from the requirement of objectivity we see that the function $g$ is isotropic, i.e.

$$
g\left(\boldsymbol{Q} \cdot \boldsymbol{D} \cdot \boldsymbol{Q}^{\mathrm{T}}, \boldsymbol{Q} \cdot \boldsymbol{M} \cdot \boldsymbol{Q}^{\mathrm{T}}\right)=\boldsymbol{Q} \cdot g(\boldsymbol{D}, \boldsymbol{M}) \cdot \boldsymbol{Q}^{\mathrm{T}}
$$

for arbitrary orthogonal tensors $Q$. If $g$ is also assumed to be linear it may be represented as

$$
g(\boldsymbol{D}, \boldsymbol{M})=a(\boldsymbol{D} \cdot \boldsymbol{M}+\boldsymbol{M} \cdot \boldsymbol{D})+b(\boldsymbol{D}: \boldsymbol{M}) \mathbf{1}+c \boldsymbol{D} \text { tr } \boldsymbol{M}
$$

in which $a, b$ and $c$ are constants.

By combining (A2.7) with the representation (A2.9) of the function $g$ we obtain a general expression for convected derivatives. By choosing particular values for the constants $a, b$ and $c$ special convected derivatives are obtained: for $a=b=c=0$, the so-called 'co-rotational' or 'Jaumann' derivative

$$
\stackrel{\leftrightarrow}{\boldsymbol{M}}=\dot{\boldsymbol{M}}-\boldsymbol{W} \cdot \boldsymbol{M}+\boldsymbol{M} \cdot \boldsymbol{W}
$$

for $a=1$ and $b=c=0$, the 'lower convected' derivative

$$
\dot{\vec{M}}=\dot{M}+L^{\mathrm{T}} \cdot \boldsymbol{M}+\boldsymbol{M} \cdot \boldsymbol{L}
$$

and for $a=-1$ and $b=c=0$, the 'upper convected' derivative

$$
\ddot{\boldsymbol{M}}=\dot{\boldsymbol{M}}-\boldsymbol{L} \cdot \boldsymbol{M}-\boldsymbol{M} \cdot \boldsymbol{L}^{\mathrm{T}}
$$

These are the most well known cases. The corresponding entrainment law is

$$
\overline{\boldsymbol{M}}(t, \tau)=\overline{\boldsymbol{R}}_{t}^{\mathrm{T}}(\tau) \cdot \boldsymbol{M}(\tau) \cdot \overline{\boldsymbol{R}}_{t}(\tau)
$$

for the co-rotational derivative. Here $\overline{\boldsymbol{R}}_{t}(\tau)$ is the 'mean rotation tensor', defined as the solution of the differential equation $(\mathrm{d} / \mathrm{d} \tau) \overline{\boldsymbol{R}}_{t}(\tau)=\boldsymbol{W}(\tau) \cdot \overline{\boldsymbol{R}}_{t}(\tau)$ with the initial condition $\overline{\boldsymbol{R}}_{t}(\tau)=\mathbf{1}$. For the lower convected derivative the corresponding entrainment law is

$$
\overline{\boldsymbol{M}}(t, \tau)=\boldsymbol{F}_{t}(\tau)^{\mathrm{T}} \cdot \boldsymbol{M}(\tau) \cdot \boldsymbol{F}_{t}(\tau)
$$

and for the upper convected derivative

$$
\bar{M}(t, \tau)=F_{r}(\tau)^{-1} \cdot M(\tau) \cdot F_{t}(\tau)^{-\mathrm{T}} .
$$

A more elaborate derivative, based upon (A2.7) and (A2.9) is the Gordon and Schowalter derivative

$$
\stackrel{\square}{\boldsymbol{M}}=\dot{\boldsymbol{M}}-\tilde{\boldsymbol{L}} \cdot \boldsymbol{M}-\boldsymbol{M} \cdot \tilde{\boldsymbol{L}}^{\mathrm{T}}
$$

with $\tilde{\boldsymbol{L}}$, an 'effective velocity gradient' defined as $\tilde{\boldsymbol{L}}=\boldsymbol{L}-\zeta \boldsymbol{D}$ (Gordon and Schowalter 1972). This corresponds to the case that in (A2.9) $a=\zeta-1$ and $b=c=0$. The corresponding entrainment law is

$$
\overline{\boldsymbol{M}}(t, \tau)=\tilde{\boldsymbol{F}}_{t}(\tau)^{-1} \cdot \boldsymbol{M}(\tau) \cdot \tilde{\boldsymbol{F}}_{i}(\tau)^{-\mathrm{T}}
$$

in which $\tilde{F}_{t}(\tau)$ is the solution of the equation $(\mathrm{d} / \mathrm{d} \tau) \tilde{F}_{t}(\tau)=\tilde{L}(\tau) \cdot \tilde{F}_{t}(\tau)$. The general case with non-vanishing $a, b$ and $c$ was used by Oldroyd (1958). We will denote this derivative by

$$
\left(\frac{\delta \boldsymbol{M}}{\delta \tau}\right)_{a b c}=\dot{\boldsymbol{M}}-\boldsymbol{W} \cdot \boldsymbol{M}+\boldsymbol{M} \cdot \boldsymbol{W}+a(\boldsymbol{D} \cdot \boldsymbol{M}+\boldsymbol{M} \cdot \boldsymbol{D})+b(\boldsymbol{D}: \boldsymbol{M}) \mathbf{1}+c \boldsymbol{D} \operatorname{tr} \boldsymbol{M}
$$


It is important to note that in continuum mechanics there is no special reason for using any particular type of convected derivative. From empirical or microscopical considerations, however, a preference for some types of convected derivatives in particular constitutive equations may be justified.

\section{References}

Abdel-Khalic S I, Hassager O and Bird R B 1974 J. Chem. Phys. $614312-16$

Acierno D, La Mantia F A, Marrucci G and Titomanlio G 1976 J. Non-Newtonian Fluid Mech. 1 125-46 Ait-Kadi A, Grmela M and Carreau P J 1988 Rheol. Acta 27 241-54

Ajji A, Carreau P J, Grmela M and Schreiber H P 1989 J. Rheol. 33 401-20

Astarita G and Marrucci G 1974 Principles of Non-Newtonian Fluid Mechanics (London: McGraw-Hill) Batchelor G K 1970 J. Fluid Mech. 41545

1977 J. Fluid Mech. 83 97-117

Bernstein B, Kearsley E and Zapas L 1963 Trans. Soc. Rheol. 7 391-410

Bird R B, Armstrong R C and Hassager O 1987a Dynamics of Polymeric Liquids Vol. 1 (New York: Wiley) Bird R B and Carreau P J 1968 Chem. Eng. Sci. 23 427-34

Bird R B, Curtiss C F, Armstrong R C and Hassager O 1987b Dynamics of Polymeric Liquids (2nd edn) vol. 2 Kinetic Theory (New York: Wiley)

Bird R B, Hassager O and Abdel-Khalik S I 1974 AICHE J. 20 1041-66

Bogue D C 1966 I.E.C. Fund. 5253

Bolder H 1969 Arch. Rational Mech. Anal. 35 321-41

Bueche F 1956 J. Chem. Phys. 25599

Carreau P J 1968 PhD Thesis University of Wisconsin, Madison 1972 Trans. Soc. Rheol. 16 99-127

Casimir H B G 1945 Rev. Mod. Phys. 17343

Coleman B D 1962 Arch. Rational Mech. Anal. 9273

Coleman B D and Noll W 1960 Arch. Rational Mech. Anal. 6355

1961 Rev. Mod. Phys. 33 239-49

Cosserat E and Cosserat F 1907 C.R. Acad. Sci., Paris $1451139-42$

Crimale W O Jr, Ericksen J L and Filbey G L 1958 Arch. Rational Mech. Anal. 1 410-17

Crochet M J, Davies A R and Walters K 1984 Numerical Simulation of Non-Newtonian Flow (Amsterdam: Elsevier)

Curtiss C F and Bird R B 1981 J. Chem. Phys. 74 2016-33

De Kee D and Carreau P J 1979 J. Non-Newtonian Fluid Mech. 6 127-43

Doeksen D K, Jongschaap R J J and Kamphuis H 1985 Polym. Eng. Sci. 25 782-7

Doi M 1983 J. Chem. Phys. 795080

Doi M and Edwards S F 1978 J. Chem. Soc., Faraday Trans. II 74 1789-832

$1979 \mathrm{~J}$. Chem. Soc., Faraday Trans. II 75 38-154

1986 Theory of Polymer Dynamics (Oxford: Oxford University Press)

Eckart C 1948 Phys. Rev. 73 373-82

Ericksen J L 1960 Kolloid Z. 173 117-22

Eringen A C 1964 Int. J. Eng. Sci. 2 205-17

Ferry J D 1980 Viscoelastic Properties of Polymers (3rd edn) (New York: Wiley)

Fixman M 1965 J. Chem. Phys. 423831

Fixman M and Kovacs J 1974 J. Chem. Phys. 61 4939, 4950

1975 J. Chem. Phys. 63935

Flory P J 1969 Statistical Mechanics of Chain Molecules (New York: Wiley-Interscience)

de Gennes P G 1971 J. Chem. Phys. 55 572-9

Geurts B J and Jongschaap R J J 1988 J. Rheol. 32 353-65

Giesekus H 1962 Rheol. Acta 2 50-62

1966 Rheol. Acta 5 29-35

_ 1982 J. Non-Newtonian Fluid Mech. 11 69-109

Goddard J D 1967 Trans. Soc. Rheol. 11381 1979 Adv. Appl. Mech. 19 143-219

Goddard J D and Miller C 1966 Rheol. Acta 5177

Gordon R J and Schowalter W R 1972 Trans. Soc. Rheol. 16 79-97 
Graessley W W 1974 Adv. Polym. Sci. 161

Green A E and Rivlin R S 1957 Arch. Rational Anal. 11

Green M S and Tobolsky A V 1947 J. Chem. Phys. 15651

Grmela M 1985 Phys. Lett. 111A 41-4

1986a Physica 21D 179-212

1986b J. Rheol. 30 707-28

Grmela M and Carreau P J 1987 J. Non-Newtonian Fluid Mech. 23 271-94

Hand G L 1962 J. Fluid Mech. 13 33-46

Huilgol R R 1978 J. Non-Newtonian Fluid Mech. 4 269-75

Irving J H and Kirkwood J G 1950 J. Chem. Phys. 18817

Johnson M W and Segalman D 1977 J. Non-Newtonian Fluid Mech. 2 255-70

1981 J. Non-Newtonian Fluid Mech. 9 33-46

Jongschaap R J J 1981 J. Non-Newtonian Fluid Mech. 8183

1987 Rheol. Acta 26 328-35

1988 Rheol. Acta 26 99-102

1989 Ann. Meeting of the Society of Rheology, Gainsville

Jongschaap R J J and Doeksen D K 1983 Rheol. Acta 22 4-11

Jongschaap R J J and Kamphuis H 1986 in Integration of Fundamental Polymer Science and Technology vol.

1 (ed. P J Lemstra and L A Kleintjens) (Amsterdam: Elsevier) p. 346

Jongschaap R J J, Kamphuis H and Doeksen D K 1983 Rheol. Acta 22539

Kamphuis H, Jongschaap R J J and Mijnlieff P F 1984 Rheol. Acta 23329

Kaye A 1962 Note No 134 College of Aeronautics, Cranfield

1966 Br. J. Appl. Phys. 17 803-6

Kirkwood J G 1949 Rec. Trav. Chim. 68649

1967 Macromolecules (New York: Gordon and Breach)

Kramers H A 1944 Physica 11 1-19

Larson R G 1981 J. Non-Newtonian Fluid Mech. 8271

1983 J. Non-Newtonian Fluid Mech. 13 279-308

- 1984 J. Rheol. 28 545-71

1988 Constitutive Equations for Polymer Melts and Solutions (Boston: Butterworth)

Leonov A I 1976 Rheol. Acta 15 85-98

Lhuillier D and Ouibrahim A $1980 \mathrm{~J}$. Mécanique 19 1-17

Lodge A S 1956 Trans. Faraday Soc. 52 120-30

1964 Elastic Liquids (New York: Academic)

Lodge A S, Armstrong R C, Wagner M H and Winter H H 1982 Pure Appl. Chem. 54 1349-59

Lodge A S and Wu Y 1971 Rheol. Acta $10539-53$

Martin P C, Parodi Q and Perhan P S 1972 Phys. Rev. A 6 2401-20

Maugin G A and Drouot R 1983 Int. J. Eng. Sci. 21 705-24

Maxwell B and Chartaff R P 1965 Trans. Soc. Rheol. 9 41-52

Meisner J 1949 Z. Naturf. a 4594

Meister B J 1971 Trans. Soc. Rheol. 15 63-89

Meixner J 1953 Kolloid. Z. 1342

Noll W 1958 Arch. Rational Mech. Anal. 2 197-226

1962 Arch. Rational Mech. Anal. 1197

Oldroyd J G 1950 Proc. R. Soc. A200 523-41

- 1958 Proc. R. Soc. A 245 278-97

1961 Rheol. Acta $1337-44$

Onsager L 1931 Phys. Rev. 37405

1932 Phys. Rev. 382265

Pearson J R A 1985 Mechanics of Polymer Processing (London: Elsevier)

Petruccione F and Biller P $1988 \mathrm{~J}$. Chem. Phys. 89 577-82

Phan-Thien N and Tanner R I 1977 J. Non-Newtonian Fluid Mech. 2 353-65

Pipkin A C 1964 Rev. Mod. Phys. 36 1034-41

Reiner M 1946 Am. J. Math. 68672

Rivlin R S 1947 Nature 160611

Rivlin R S and Sawyers K N 1971 Ann. Rev. Fluid Mech. 3 117-46

Rouse P E 1953 J. Chem. Phys. 211272

Soong D S and Shen M 1979 J. Polym. Sci. Polym. Lett. 17 595-9

Spencer A J M and Rivlin R S 1959 Arch. Rational Mech. Anal. 2 309-36 
1960 Arch. Rational Mech. Anal. 4 214-30

Stickforth J 1981 Int. J. Eng. Sci. 19 1775-88

1986 Rheol. Acta 25 447-58

Tanner R I 1983 J. Appl. Mech. $501181-90$

1985 Engineering Rheology (New York: Oxford University Press)

Truesdell C and Noll W 1965 Non-linear Field Theories of Mechanics (Springer Encyclopedia of Physics III/3) (Berlin: Springer)

Wagner M H 1976 Rheol. Acta 15 136-42

1979 Rheol. Acta 18 33-50

Wagner M H and Stephenson S E 1979 J. Rheol. 23 491-504

Walters K 1965 Rheometry (London: Chapman and Hall)

Wax N 1954 Noise and Stochastic Processes (New York: Dover)

White J L and Metzner A B 1963 J. Appl. Polym. Sci. 7 1867-89

Wiegel F W 1969 Physica 43 33 44

Wiegel F W and De Bats F Th 1969 Physica 43 33-44

Yamamoto M 1956 J. Phys. Soc. Japan 11413

Yasuda K, Armstrong R C and Cohen R E 1981 Rheol. Acta 20 163-78

Zahorski S 1982 Mechanics of Viscoelastic fluids (The Hague: Martinus Nijhoff)

Zimm B H 1956 J. Chem. Phys. 24269 